\title{
Event-Driven ECG Sensor in Healthcare Devices for Data Transfer Optimization
}

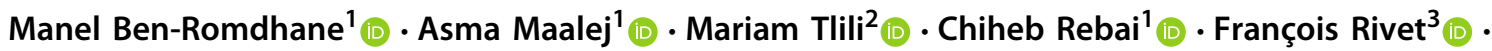 \\ Dominique Dallet ${ }^{3}$
}

Received: 26 July 2019 / Accepted: 19 March 2020 / Published online: 31 March 2020

(c) King Fahd University of Petroleum \& Minerals 2020

\begin{abstract}
The long-term monitoring of cardiovascular signs requires a wearable and connected electrocardiogram (ECG) healthcare device. It increases user's comfort and diagnosis quality of chronic cardiac and/or high-risk patients. This paper covers the enormous data to be transmitted from the ECG device to the physician's, namely the cardiologist's, control unit. Existent ECG devices uniformly sample analog signals and convert them to digital samples which are compressed before data transmission. However, event-driven sampling simultaneously compresses and samples. Therefore, this paper quantitatively compares successive approximation register analog-to-digital converter (SAR ADC) with discrete wavelet transform (DWT) compression and level-crossing analog-to-digital converter (LC-ADC). Evaluation metrics are the percent root-mean-square difference (PRD), bit compression ratio (BCR) and data length in bits. When a 12-bit reconstruction is operated on the outputs of an 8-bit LC-ADC with 12-bit and 10-kHz reference counter, the BCR is equal to $80 \%$ for $75 \%$ of test ECG signals. That is better than the $71.87 \%$ BCR of the 12-bit 1-kHz SAR ADC with DWT compression. The modeled LC-ADC guarantees a signal quality in terms of PRD comparable to the PRD of the SAR ADC with DWT compression. The data length in bits of the LC-ADC is lower than the data length in bits of the SAR ADC with more than 14-bit resolution with DWT compression for $82 \%$ of the test ECG signals. However, for lower resolutions, to obtain lower power consumption for radiofrequency transmission, a better alternative remains the SAR ADC with DWT compression.
\end{abstract}

Keywords Analog-to-digital converter · Event-driven sampling · Successive approximation of registers · ECG sensor · Discrete wavelet transform $\cdot$ Data compression

\section{Introduction}

Manel Ben-Romdhane

manel.benromdhane@ supcom.tn

Asma Maalej

asma.maalej@supcom.tn

Mariam Tlili

maryem.tlili@supcom.tn

Chiheb Rebai

chiheb.rebai@supcom.tn

François Rivet

francois.rivet@ims-bordeaux.fr

Dominique Dallet

dominique.dallet@ims-bordeaux.fr

1 COSIM Research Laboratory, SUP'COM, University of Carthage, Cité Technologique des Communications, 2083 El Ghazala, Ariana, Tunisia

2 LIP6, CNRS, Sorbonne University, 75005 Paris, France
In health care, there are considerable developments in medical devices in order to provide continuously or on-demand monitoring about the well-being of the chronic cardiac and/or high-risk patients [1]. There are also reported works on electrocardiogram (ECG) biometric mode for the identification and authentication of individuals but also aiming at securing data and accessing services, as medical ones [2, 3]. Additionally, American Heart Association (AHA) statistics show that the coronary heart disease is the leading cause of death in the USA. In fact, $43.8 \%$ of deaths are caused by cardiovascular diseases (CVDs) [4]. Therefore, Internet of things (IoT) healthcare devices, namely electrocardiogram (ECG)

3 IMS Research Laboratory, University of Bordeaux, Bordeaux INP Enseirb-Matmeca, 351 Cours de la Libération, Bâtiment A31, 33405 Talence Cedex, France 
sensors, become increasingly vital to the twenty-first century patients. Increased need for ECG devices is driven due to rising healthcare costs and increasing rates of CVD worldwide. These factors drive the demand for low-cost, reliable and wearable ECG devices. These devices allow ECG signal acquisition [5], digital signal processing [6] and data transmission to the remote physician, namely the cardiologist [1], for abnormal ECG signal analysis [1]. This leads the authors to focus on ECG signal acquisition but not only ECG features extraction. Commercialized wearable ECG devices as QARDIOCORE by Qardio [7] and wired ECG devices derive from 1 to 12 signals, known as leads in medical context. Each is composed of the $\mathrm{P}$ wave, the QRS complex and then the $\mathrm{T}$ wave.

Currently, the most recent wearable ECG devices are KardiaMobile by AliveCor [8], QARDIOCORE by Qardio [9] and Apple Watch Series 4 by Apple [8]. KardiaMobile records a single lead from $30 \mathrm{~s}$ to $5 \mathrm{~min}$ to detect atrial fibrillation, bradycardia, tachycardia as well as normal heart rhythm. Apple Watch Series4 also records a single-lead ECG for $30 \mathrm{~s}$ and detects only atrial fibrillation or normal heart rhythm. QARDIOCORE, meanwhile, detects heart rate variability without diagnosis prediction. It records 3 ECG signals or leads with a 16-bit analog-to-digital converter (ADC) at 600$\mathrm{Hz}$ sampling frequency. It sends a message via Bluetooth wireless communication to the connected smartphone. Then, the smartphone transfers the message to the physician within few seconds. KardiaMobile's ADC samples at $300 \mathrm{~Hz}$, then quantizes and codes each sample on 16 bits. No additional information is available regarding Apple Watch Series4's sampling frequency and ADC resolution.

As an example, the KardiaMobile's analog-to-digital converter (ADC) delivers a 4.8-kbits message during one second of conversion for a single signal. In the scientific literature, to optimize or reduce data transfer, decrease sensors' power consumption and increase wireless sensor network lifetime, three research axes have been developed. First, communication protocols consider the optimization of data communication between the healthcare sensors or devices, the patient's smartphone and the hospital server [10]. Second, radiofrequency $(\mathrm{RF})$ transceiver is miniaturized to realize a comfortable wearable smart sensor as in [11]. Third, message data length of the ECG is reduced in order to optimize the data transfer via a given wireless communication transceiver [12]. In this context, this paper argues that the event-driven ECG sensor should be used to reduce message data length and to optimize data transfer.

Thus, it is interesting to reduce the message data length in bits, which is obtained at the ADC output, to optimize data transfer power consumption [13]. An Lb-bit message is transmitted during a given time duration from the ADC output to the receiver. The transmitter's data transfer power consumption is consequently Lb multiplied by the wireless transmission power consumption per bit [10, 14]. Besides, the message data length in bits during one second is the message to be transferred during one second and not the wireless communication standard's throughput.

As far as ECG signal acquisition is concerned, the wearable ECG devices and many wired or wireless ECG acquisition systems use generally Nyquist ADCs and rarely oversampling ADCs. Data are uniformly extracted at every sampling period, considering at least Nyquist frequency according to Shannon theorem [5]. In fact, sigma-delta ADCs offer higher resolutions than Nyquist ADCs, but generally require higher power consumption due to their oversampling technique. Besides, the maximum frequency of ECG signal is $250 \mathrm{~Hz}[15,16]$. Consequently, the sampling frequency must be higher than $500 \mathrm{~Hz}$ according to Shannon theorem. ADC survey shows the successive approximation register ADCs (SAR ADCs) are more suitable for ECG signal acquisition than pipeline, continuous-time sigma-delta (CTSD), switched-capacitor sigma-delta (SCSD) and flash ADCs according to the signal-to-noise and distortion ratio (SNDR) and for a given technology as the $180 \mathrm{~nm}$ complementary metal oxide semiconductor (CMOS) [17]. Moreover, SAR ADC architectures use few building blocks and thus consume less power than other ADC architectures [18]. Their propagation delays are lower than those of other architectures and are less sensitive to technology accuracy limits of passive components and jitter.

However, many ADCs need data compression to send or store lower amount of data that represent original samples. The aim is to restore the ECG signal after decompression at the distant ECG control unit [5]. The obtained ECG signal is quasi-identical to the original ECG signal. It is then analyzed by the physician who sends the diagnosis to the patient. Knowing that, off-line QRS complex detection [19], realtime QRS complex detection or even ECG features extraction [1] methods are irreversible compression methods. Indeed, they do not allow to recover the ECG signal. Conversely, in the literature, some proposals are made with event-driven ECG sensors that sample signals regarding levels as in [20]. These sensors require level-crossing analog-to-digital converter (LC-ADC) that combines event-driven sampling and compression simultaneously.

This paper aims at selecting the best-fit discrete wavelet transform (DWT) to compress a successive approximation register analog-to-digital converter (SAR ADC) output in an ECG sensor and to fulfill a quantitative comparative study with the level-crossing ADC (LC-ADC). To the best of the authors' knowledge, no quantitative comparative study between (A1) the SAR ADC architecture which is followed by a DWT compression stage and (A2) the LC-ADC architecture has been conducted. Thus far, this paper's main contribution is to achieve a quantitative comparison by: 
- Reviewing on the one hand, the literature of the ADCs that relies on the SAR ADC as the best candidate among Nyquist besides sigma-delta ADCs, and the LC-ADC as the event-driven sampling-based ADC, and on the other, the literature of the data compression methods which decides on DWT compression method,

- Simulating examples of the architectures (A1) and (A2) where test ECG signals from databases [21] are applied at their inputs,

- Comparing the percent root-mean-square differences (PRDs), the bit compression ratios (BCRs) and the message data lengths in bits of (A1) and (A2),

- Selecting the best architecture to reduce the message data length in bits and save power in wireless sensor networks.

Consequently, the remaining of this paper is organized as follows:

- Section 2 gives a state of the art of ECG signal acquisition and data compression.

- Section 3 details the performance evaluation test-bench steps of (A1) the SAR ADC with discrete wavelet transform (DWT) compression and states how to select the most suitable DWT to compress the SAR ADC output. It also briefs (A2) the LC-ADC, its performance evaluation test bench, the data transfer optimization and evaluation metrics that are required to compare (A1) and (A2).

- Section 4 presents and discusses the quantitative comparison of the evaluation metrics as the percent rootmean-square differences (PRD), the bit compression ratios (BCR) and the data length in bits. The objective is to provide a defendable selection of (A1) the SAR ADC with DWT compression or (A2) the LC-ADC.

- Section 5 summarizes the whole paper and draws future works.

\section{ECG Signal Acquisition and Data Compression}

In all manufactured electrocardiogram (ECG) acquisition devices and most ECG acquisition devices in the literature, the analog-to-digital converter (ADC) uniformly samples and quantizes ECG analog signals to obtain ECG data, $\mathrm{ECG}_{\text {out }}$. In an ECG signal, the waveform from the $\mathrm{T}$ wave to the $\mathrm{P}$ wave has usually a lower amplitude and a larger duration than those of the $\mathrm{P}$ wave, the QRS complex and the T wave [22]. Thus, data compression of the uniform ECG data is required to reduce the message data length to be transferred from the ECG device to a distant control unit for an ECG diagnosis. However, this paper also deals with the sampling regarding analog signal amplitude events. This signal acquisition is indeed more suitable for the ECG signal. In this section, the
ECG monitoring system architecture is introduced to show its key blocks. Then, the authors present a review of ECG signal acquisition, namely the successive approximation register ADCs (SAR ADCs) and level-crossing ADCs (LC-ADCs). Finally, existent data compression methods that follow a SAR ADC are provided. They are compared in terms of bit compression ratio (BCR) in order to evaluate ECG data reduction and percent root-mean-square difference of data compression $\left(\mathrm{PRD}_{\text {comp }}\right)$ that evaluates the recovered-signal quality.

\subsection{ECG Monitoring System Architecture}

The configuration of an ECG monitoring system, as presented in Fig. 1, is composed of the wearable ECG sensor, the signal acquisition, the data compression and the distant ECG control unit. The obtained ECG signal from two electrodes is filtered, amplified then sampled and quantized in the analog front end to deliver the digitized signal. In commercialized systems [1], ECG signal acquisition is only operated using Nyquist or sigma-delta ADCs. However, in the ECG devices that have been suggested in the literature [20], level-crossing ADCs have been also employed. The compression is required for Nyquist or sigma-delta ADCs but never quantitatively compared to the inherent compression of level-crossing ADCs. In fact, the LC-ADCs decrease the amount of data compared to Nyquist ADCs and considerably decrease the amount of data compared to sigma-delta ADCs. After compression or source encoding, followed by radiofrequency $(\mathrm{RF})$ transmission, the digital reconstruction block performs signal decompression or source decoding. In case of event-driven devices, interpolation is also required to recover uniform samples. The control unit manages the signal decompression, the digital signal processing and the interpolation. The physician also analyzes the ECG signals using one of his communication devices and saves only received data if required.

\subsection{ECG Analog-to-Digital Converters' State of the Art}

Most of ECG acquisition systems generally digitize analog signals with successive approximation register ADC (SAR $\mathrm{ADC}$ ) as stated in the commercialized systems or in the scientific literature proposals [1]. Indeed, the SAR ADC structure is simply composed of a sample-and-hold $(\mathrm{S} / \mathrm{H})$ circuit, a comparator to compare the sample to the previous estimation, successive approximation of registers (SARs) and a digital-to-analog converter (DAC) [18]. The DAC delivers the analog value of the previous estimation. The SAR ADC converts an analog ECG signal into digital samples. Thus, the T-to-P ECG waveform, which amplitude variations are lower than $0.1 \mathrm{mV}$ during at least $0.3 \mathrm{~s}$, and the QRS complex, which amplitude is around $1 \mathrm{mV}$ during at most $0.1 \mathrm{~s}$ 

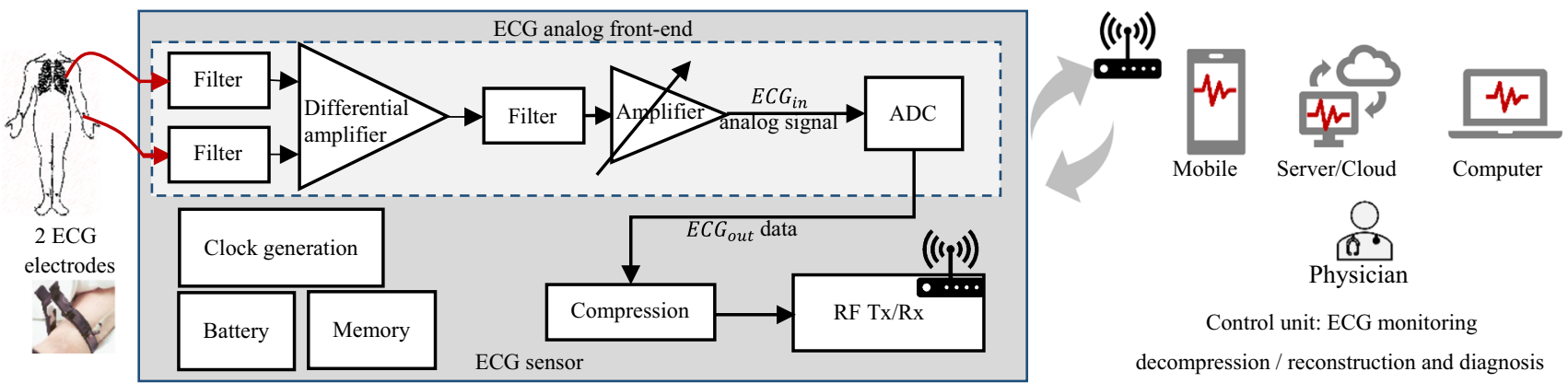

Fig. 1 Configuration of a wearable ECG monitoring system

[22], are sampled at the same frequency [23, 24]. That delivers an important number of redundant samples in the T-to-P ECG waveform. However, sampling only when events occur makes it possible to reduce the T-to-P ECG data. For instance, in the pacemaker proposal in [25], flexible resolution SAR ADC with asynchronous dynamic logic samples the ECG leads and their features after the QRS complex extraction in the analog domain.

Moreover, in [14], to reduce the data length when sampling an ECG signal with a SAR ADC, the QRS complex is sampled at a frequency higher than the sampling frequency of the remaining parts of the ECG signal. Further compression reduces the number of bits per sample. In fact, after the SAR $\mathrm{ADC}$, a digital adaptive controller is required to compare the difference between successive samples and the predefined threshold to select adequate sampling frequency. The SAR ADC parameters given in [14] are measured only for a test ECG signal. Another SAR ADC-based proposal is to sample the ECG signal at two different sampling frequencies which are selected according to its analog slope extraction [26]. Additionally, authors in [27] propose a high-precision ECG sensor system due to an ADC that integrates a stochastic flash ADC with a digitally controlled variable threshold comparator into a SAR ADC. In addition, a dynamic tracking SAR ADC and a hybrid SAR-sigma-delta ADC are proposed in $[28,29]$, respectively. Otherwise, sigma-delta converters are rarely used as in [29, 30]. Indeed, they offer higher resolutions, but require important sampling frequencies due to their oversampling technique [17].

Therefore, some of the most recent SAR ADCs are summarized in Table 1 in reference to different resolutions, $m$, that are sorted from low to high values. In fact, there are proposals to reduce the data length of the SAR ADC output $[14,24-26,28]$ and others to increase SAR ADC resolutions $[27,29]$. For the data length reduction, the SAR ADC in [28] uniformly samples the ECG signals, and then, a digital signal processing is operated. The SAR ADC in [14] samples the signals after a frequency digital selection, whereas the SAR ADC in [26] samples the signals after a frequency analog selection. Other parameters are used to compare SAR ADCs.
They are the frequency of the successive approximation of registers (SARs), $F_{\mathrm{SAR}}$, the sample-and-hold frequency or the reference frequency, $F_{\text {ref }}$, the dynamic range, the complementary metal oxide semiconductor (CMOS) technology, the power consumption and the core area, and the effective number of bits (ENOB) is presented in Table 1. The ENOB is given in the reference or is extracted from the signal-to-noise and distortion ratio (SNDR) in decibels (dB) as in (1) [27].

$\mathrm{ENOB}=\frac{\mathrm{SNDR}-1.76}{6.02}$

From the SAR ADC state of the art, the outputs require data length reduction by either modifying the SAR ADC architecture or applying a compression technique at the SAR ADC output.

Therefore, the event-driven signal acquisition is a more appealing architecture than the SAR ADC, also known as level-crossing sampling. It has been introduced since the 1980s as a combined compression digital encoding technique of analog signals [31], but its circuit implementation or modeling is recent. In fact, level-crossing analog-todigital converter (LC-ADC) architectures are proposed to sample signals regarding amplitude levels instead of time instants. That detects the exact amplitude values instead of the quantized data values. The LC-ADC thus guarantees the conversion of the samples at selected levels. However, it requires a quantization of each interval between two successive level-crossing instants. In the last decade, for instance, authors in [32] and [33] studied event-driven speech and biomedical signals, respectively. Different circuit realizations were particularly proposed for bio-signal acquisitions, ranging from LC-ADCs in application-specific integrated circuits (ASICs) $[34,35]$ to fully integrated system-on-chip (SoC) solutions for wearable sensors [20].

As far as LC-ADC architecture types are concerned, some LC-ADC architectures use the fixed-window level-crossing type [35-37], whereas others use the floating-window type $[20,38,39]$. In fact, the floating-window type is the conventional and the widely adopted type in LC-ADC architectures. It consists of two comparators, two digital-to-analog convert- 


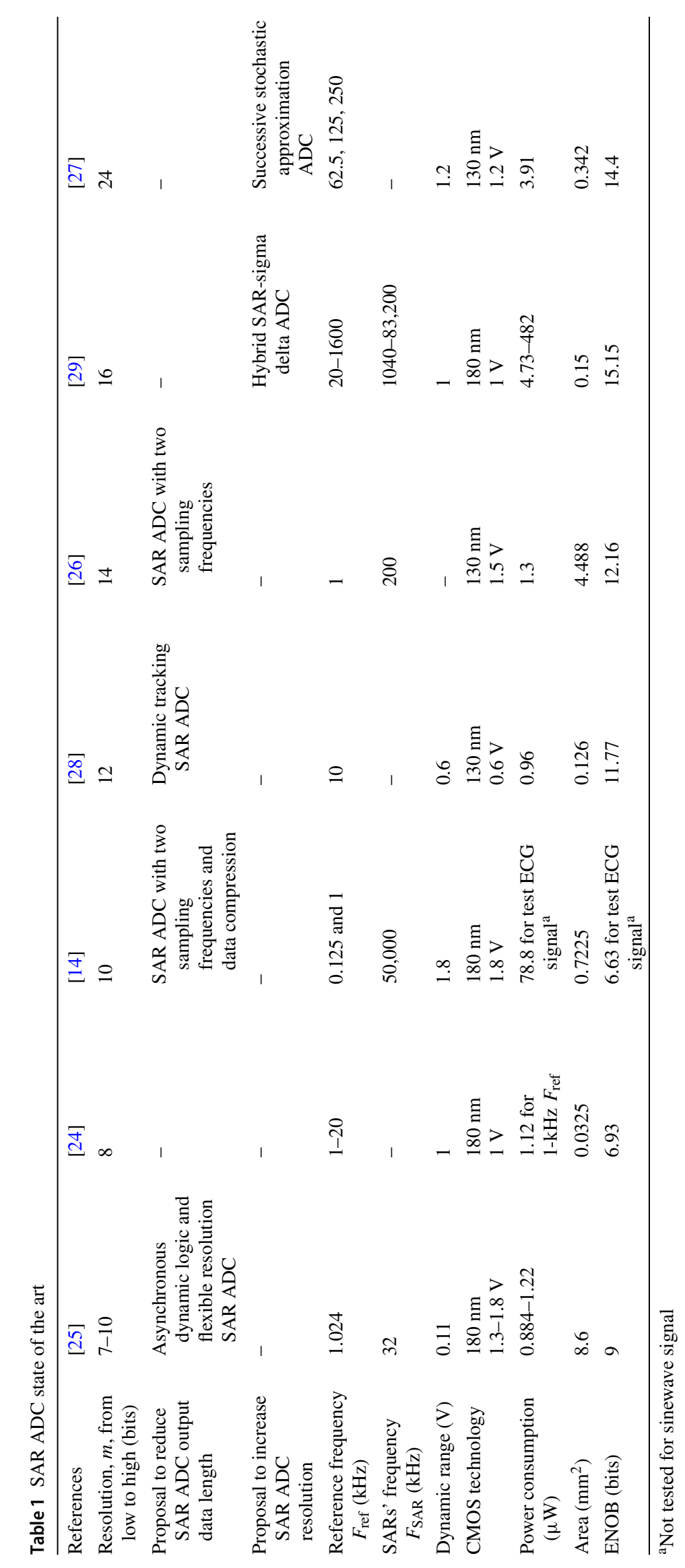

(1) 몸inger 
ers (DACs), a logic control and a reference counter clock. First, the comparators detect the input crosses of the ana$\log$ levels. Second, the DACs create analog levels bounding the input. Third, the logic control obtains up/down slope and evaluates the samples. Fourth, the reference counter clock quantizes the interval between two successive levelcrossing instants. Alternatively, the fixed-window type does not require the updating of the window between two successive analog levels, but it requires the subtraction of the DAC output from the analog input. The obtained difference is compared to the analog fixed levels. Instead of DACs in the LC-ADC, some proposals are based on a scaler to select analog levels for the comparators [40]. Furthermore, authors in $[35,38]$ suggest other solutions with variable resolutions regarding slope threshold which is the window out of the quantized time between two successive samples.

The LC-ADC state of the art is presented in Table 2. The LC-ADC references are organized according to the LC-ADC resolutions, $M$, that are sorted from 5 to 8 bits. These values are adequate to digitize the test ECG signals according to the literature. In addition, the reference counter frequency, $F_{\mathrm{C}}$, quantizes the time between two successive level-crossing instants. In Table 2, the power consumption and core area values are sometimes proportionally computed from other values in the given reference or in a previous reference of the same authors. Table 2 shows that LC-ADC power consumptions vary from 0.0024 to $106 \mu \mathrm{W}$ for test sinewave input signals at different frequencies from $1 \mathrm{~Hz}$ to $10 \mathrm{kHz}$. These values are close to SAR ADCs' values from 0.884 to $78.8 \mu \mathrm{W}$ when $1 \mathrm{kHz}$ reference frequency is adopted. However, it is important to mention that for low-frequency and low-amplitude inputs, as in T-to-P ECG waveform, the LCADC samples less densely in time than for high-frequency and high-amplitude inputs, as in QRS complex. As far as the areas are concerned, they are generally similar because most of SAR ADC and LC-ADC architectures are based on comparators, DACs and digital processing.

From the current review of the literature, tested circuits and measurement results with a test ECG signal are rarely published. The authors give in SAR ADC state of the art in Table 1, the measured results of [14] for test ECG signal, and in LC-ADC state of the art in Table 2, measured results of [20] are given for $1 \mathrm{kHz}$ sinewave signal. ECG sensor in [20] is tested with an ECG signal, but performances are not measured. In fact, the ENOB of [14] is 6.63 bits for a 10-bit SAR ADC, $1 \mathrm{kHz}$ reference frequency and test ECG signal, whereas it is 7.43 bits [20] for an 8-bit LC$\mathrm{ADC}$ with a sinewave test signal at the input. That ENOB is estimated from SNDR which is computed according to the ECG wireless sensor's input referred noise and input voltage. Therefore, LC-ADC and SAR ADC have almost the same performances leading to similar sensitivity levels $[14$, $20,35]$. Thus, aside from the combined signal acquisition

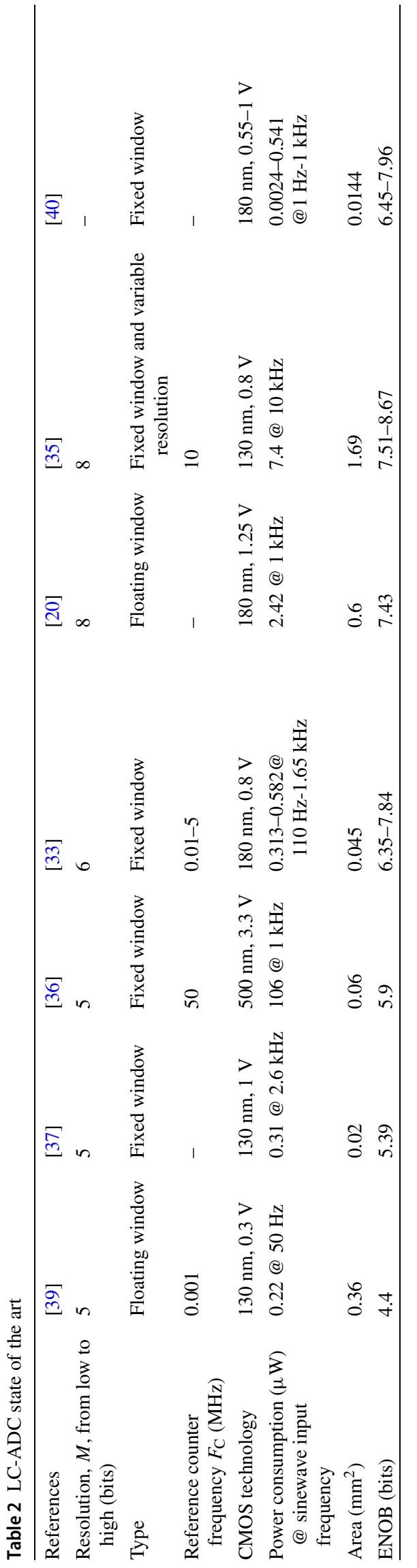


and inherent compression of analog signals with LC-ADC, many compression techniques have been proposed in the literature. These techniques were implemented and tested on digital test ECG data at the SAR ADC outputs. In the next subsection, the existent compression methods are introduced and compared.

\subsection{ECG Data Compression Methods' State of the Art}

Long-term monitoring using ECG electrodes connected to smartphones and/or point-of-care devices has to save and/or send selected ECG data for an early detection of cardiovascular diseases (CVDs). Since 1968, many works have been published covering ECG data compression, redundancy reduction and parameters' extraction. For example, in ECG acquisition systems, data reduction for the sake of storage is introduced in [41] with amplitude zone time epoch coding (AZTEC). In fact, large amount of ECG data compression is applied either to store or to transmit less data in order to reduce bit rate and time processing while decreasing power consumption [42]. In the present paper, the uniform ECG data, $\mathrm{ECG}_{\text {out }}$, are compressed to obtain the data compression output, $\mathrm{ECG}_{\mathrm{comp}}$. To evaluate data compression methods, it is required to measure the data lengths in bits during the same time duration, DT, before and after the data compression. Thus, the bit compression ratio, BCR, is computed as given in (2),

$\operatorname{BCR}(\%)=100 \times \frac{m L-\mathrm{Lb}_{\text {comp }}}{m L}$

where $m$ is the SAR ADC resolution, $L$ is the number of samples, $\mathrm{ECG}_{\mathrm{out}}$, and $\mathrm{Lb}_{\text {comp }}$ is the data length in bits of $\mathrm{ECG}_{\mathrm{comp}}$. The lower the data length in bits after compression is, the higher the BCR of the data compression method is.

Decompression quality can be clinically acceptable although many techniques highly compress ECG data. In this paper, the presented methods are applied after the SAR ADC and require ECG data decompression to recover estimated ECG data. From the recovered ECG signal, the percent root-mean-square difference of data compression, $\mathrm{PRD}_{\text {comp }}$, is assessed, and the physician accomplishes the diagnosis as explained in Fig. 1. Indeed, to evaluate ECG data compression quality, the $\mathrm{PRD}_{\text {comp }}$ is computed as in (3) [43],

$\operatorname{PRD}_{\text {comp }}(\%)=100 \times \sqrt{\frac{\sum_{i=1}^{L}\left(\mathrm{ECG}_{\text {out }}(i)-\widehat{\mathrm{ECG}}_{\text {out }}(i)\right)^{2}}{\sum_{i=1}^{L}\left(\mathrm{ECG}_{\text {out }}(i)-\overline{\mathrm{ECG}}_{\text {out }}\right)^{2}}}$

where $\mathrm{ECG}_{\text {out }}$ is the original uniform ECG data, $\widehat{\mathrm{ECG}}_{\text {out }}$ is the estimated data after decompression from $\mathrm{Lb}_{\text {comp }}$ bits, $\overline{\mathrm{ECG}}_{\text {out }}$ is the mean of the original uniform ECG data and $L$ is the original uniform ECG data length or the number of samples. In order to achieve a correct clinical diagnosis only from the $\mathrm{Lb}_{\text {comp }}$ bits [44], at most a $9 \% \mathrm{PRD}_{\text {comp }}$ is allowed. A $0 \% \mathrm{PRD}_{\text {comp }}$ reflects a lossless compression and a reversible decompression which means that $\widehat{\mathrm{ECG}}_{\text {out }}$ is exactly equal to the original uniform ECG data, $\mathrm{ECG}_{\text {out }}$.

Thus, the commonly used compression algorithms are compared in order to select the adequate compression method regarding $B C R$ and $\mathrm{PRD}_{\text {comp }}$ aiming at the evaluation of ECG data compression quality. Once the $\mathrm{PRD}_{\text {comp }}$ is lower than 9\%, the lower the ECG data length in bits after compression, $\mathrm{Lb}_{\text {comp }}$, is, the higher the $\mathrm{BCR}$ is and the better the data compression method is. The data compression methods are commonly distinguished in lossless and lossy methods. In fact, with lossless compression methods, reversible decompression algorithms recover the exact original uniform ECG

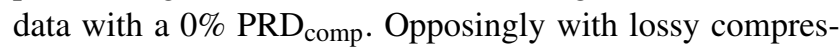
sion methods, irreversible decompression algorithms remove some samples or bits from original uniform ECG data, and others project them given a transform function and remove some of the obtain coefficients. Consequently, ECG data recovery leads up to data distortion which generates a nonzero PRD comp $_{\text {. }}$

As far as the lossless data compression methods are concerned, entropy coding is widely used with differential pulse-code modulation (DPCM) regarding predefined error threshold with prediction or interpolation [45]. In [46], the prediction with DPCM is the least complex, and the prediction with more complex algorithms, such as neural network, auto-regression filters or Lempel-Ziv, is unsuitable for realtime transmission. Besides, entropy coding algorithms, such as Huffman, arithmetic, Golomb, Golomb-Rice (GR) and fixed-length packaging, are the commonly used ones. Adopting the lossless compression methods recovers the exact original ECG data, but low BCRs are computed. Combining predictor with entropy coding algorithms makes the bit compression ratios vary between almost $48 \%$ and $58 \%$ [46-49] as shown in Table 3. Combining fuzzy optimization and Huffman region coding offers a BCR of $64.7 \%$ [50]. Nonetheless, adaptive trending predictor with modified Huffman and GR coding offers a BCR of $62.5 \%$ [51]. In these references, the obtained results are measured for a small number of test ECG signals or for test ECG signals from the same database, generally the MIT-BIH arrhythmia database [21].

However, nowadays, researchers in the field of smart health sensors adopt higher bit compression ratios with lossy data compression methods that offer a small ECG data distortion. In fact, works in [44, 52-54] are based on thresholding-based wavelets or discrete wavelet transform (DWT) as lossy compression methods. The best trade-off combines DWT with shrinkage, Huffman and run-length encoding (RLE) algorithms to offer a 96\% BCR and 3\% 
Table 3 Lossless data compression methods' state of the art

\begin{tabular}{ll}
\hline Lossless data compression method & BCR (\%) \\
\hline Linear predictor/Huffman coding [47] & 47.9 \\
Delta predictor/Golomb-Rice (GR) coding [46] & 57.9 \\
Adaptive predictor/Huffman coding [48] & 58.8 \\
Adaptive linear predictor/fixed-length packaging [49] & 56.1 \\
Fuzzy optimization/Huffman region coding [50] & 64.7 \\
$\begin{array}{l}\text { Adaptive trending predictor/modified Huffman and GR } \\
\text { coding [51] }\end{array}$ & 62.5 \\
\hline
\end{tabular}

$\mathrm{PRD}_{\text {comp }}$ [53]. Other lossy compression methods are proposed in $[42,55]$. They offer around $77 \%$ and $87 \%$ of BCR with an excellent $\mathrm{PRD}_{\text {comp }}$, that is, lower than $2 \%$. In [55], the method consists in ECG data decimation by a fractional factor. In [42], the method uses Fun algorithm that is combined with an on-demand residual Huffman coding. Thus, Huffman compression is only applied on the residual data after online decompression and residual data request.

As far as [56] is concerned, the proposed compression method detects turning angles larger than a threshold. In fact, the useful information for the diagnosis is in the rapid variations of the $\mathrm{P}$ wave, the QRS complex and the $\mathrm{T}$ wave. Another proposal comes in [12] to reach a $99 \%$ BCR, but only an $8 \%$ mean $\mathrm{PRD}_{\text {comp }}$ is obtained for all test ECG signals of MIT-BIH arrhythmia database. In fact, convolutional auto-encoder is applied to ECG data compression by adopting a spindle structure to extend and then reduce the data length. A more recent work shows a double exponential wavelet design that is combined to compressive sensing [57]. This compression method tries to reach a $90 \%$ BCR and a $6.5 \% \mathrm{PRD}_{\text {comp }}$, but these are mean values obtained from 20 test ECG signals. Additionally, in [58], the compression algorithm begins by segmentation of the test ECG signal into clinically relevant parts (the QRS complexes and possibly $\mathrm{P}$ and $T$ waves) and nonclinically relevant parts (the remaining parts of the ECG signal). The first group is compressed with differential pulse-code modulation (DPCM) and run-length encoding (RLE) that are lossless methods, whereas others are compressed due to DWT with threshold which is a lossy method.

Table 4 summarizes the lossy data compression state of the art. The obtained results are measured for one test ECG signal [56], two test ECG signals [58] or all test ECG signals [12] from MIT-BIH arrhythmia database. Thus, after the SAR ADC, the DWT-based compression methods provide the best trade-off between BCR and $\mathrm{PRD}_{\text {comp }}$ [53]. In addition, the DWT compression as implemented in [53] does not require test ECG signal segmentation as in [58], and decompression is always irreversible. However, the LC-ADC seems to be appealing as it simultaneously operates ECG signal acquisition and data compression. Besides, the reconstruction is
Table 4 Lossy data compression methods' state of the art

\begin{tabular}{lll}
\hline Lossy data compression method & BCR (\%) & PRD $_{\text {comp }}(\%)$ \\
\hline $\begin{array}{l}\text { Simultaneous orthogonal matching } \\
\text { pursuit [52] }\end{array}$ & 86.1 & 2.57 \\
$\begin{array}{l}\text { Compressive sensing [44] } \\
\text { DWT with shrinkage [53] }\end{array}$ & 71 & 9 \\
$\begin{array}{l}\text { DWT with shrinkage/Huffman/RLE } \\
\text { [53] }\end{array}$ & $65-96$ & 0.12 \\
$\begin{array}{l}\text { Two sampling frequencies and } \\
\text { Huffman encoding [14] }\end{array}$ & 64 & 0.82 \\
$\begin{array}{l}\text { DWT/RLE with modified threshold } \\
\quad \text { [54] }\end{array}$ & 81 & 2.7 \\
$\begin{array}{l}\text { Decimating by a fractional factor [55] } \\
\text { Fan algorithm/on-demand residual }\end{array}$ & 77.7 & 0.53 \\
$\quad$ Huffman coding [42] & 87.1 & 0.51 \\
$\begin{array}{l}\text { Turning-angle-based compression [56] } \\
\text { Spindle convolutional auto-encoder }\end{array}$ & $71.08^{\mathrm{a}}$ & $5.87^{\mathrm{a}}$ \\
$\quad$ [12] & 99.06 & $2.453-18.2$ \\
$\begin{array}{l}\text { Compressive sensing and double } \\
\text { exponential wavelet transform [57] }\end{array}$ & $10-90^{\mathrm{b}}$ & $0.43-6.5^{\mathrm{b}}$ \\
$\begin{array}{l}\text { DPCM/RLE or DWT with threshold } \\
\text { according to the part of ECG waves } \\
\text { [58] }\end{array}$ & $93^{\mathrm{a}}$ & $2.81^{\mathrm{a}}$ \\
\hline
\end{tabular}

${ }^{\text {a Record }} 117$ from MIT-BIH arrhythmia database

${ }^{b}$ Mean values for 20 ECG signals from MIT-BIH arrhythmia database ${ }^{\mathrm{c}}$ Record 110 from MIT-BIH arrhythmia database

always irreversible. In fact, although the LC-ADC allows lower amount of data regarding the SAR ADC with DWT compression, quantitative comparative study has not been conducted yet in terms of bit compression ratio, message data length in bits versus signal quality for both architectures. Thus far, the main contribution of this paper is a quantitative comparison between (A1) the SAR ADC architecture with DWT compression and (A2) the LC-ADC architecture. This comparison is important as the lower the message data length in bits is, the lower the ECG sensor power consumption of $\mathrm{RF}$ transmission is. In fact, the data length in bits is multiplied by the RF transmission power consumption per bit [14] to obtain the ECG sensor power consumption, knowing that RF transmission results in $63 \%$ of the power consumption of a given ECG sensor [13]. Thereafter, the next section describes the evaluation methods for both architectures and defines the evaluation metrics.

\section{Evaluation Methods}

As provided previously in Table 1, recent SAR ADC architectures add specific compression as in [27,29] or are combined with a further data compression block. Both must reduce the large amount of data delivered by the SAR ADC as in [25] to reduce the data amount to be sent to the control unit. This sec- 
tion deals with evaluation methods that are required for the quantitative comparison between (A1) the SAR ADC with discrete wavelet transform (DWT) compression and (A2) the LC-ADC. First, the authors acquaint the SAR ADC architecture. The performance evaluation test bench of SAR ADC that is followed by DWT compression is also proposed to test (A1) with many test ECG signals at its input. The DWT is inherently reversible transform. Opposingly, DWT compression is irreversible, and it is a lossy compression. Some of the obtained DWT output coefficients are removed before data transmission to the distant ECG control unit. Second, DWT-based ECG data compression is studied, and the most adequate DWT compression is proposed. Third, (A2) the designed LC-ADC architecture alongside its performance evaluation test bench is introduced. Moreover, optimization of the LC-ADC outputs is stated to reduce the data transfer in healthcare devices. Forth, test ECG signals from Physionet are presented together with the required evaluation metrics to analyze the quantitative comparison of (A1) the SAR ADC with DWT compression and (A2) the LC-ADC.

\subsection{SAR ADC with DWT Compression Evaluation}

The SAR ADC first samples and holds $(\mathrm{S} / \mathrm{H})$ the analog input signal, $\mathrm{ECG}_{\mathrm{in}}$, at the $\mathrm{S} / \mathrm{H}$ frequency or the reference frequency, $F_{\text {ref }}$. Second, the obtained sample is compared to the first analog approximated value. This value is delivered after the successive approximation registers (SARs) and the digital-to-analog converter (DAC) [14]. Each cycle takes $1 / F_{\mathrm{SAR}}$ where the SARs' frequency, $F_{\mathrm{SAR}}$, is at least $m$ times the reference frequency, $F_{\text {ref }}$. The SARs' initial value is set with the most significant bit (MSB) at " 1 " and other $m-1$ bits at "0" where $m$ is the SAR ADC resolution. The DAC output is then equal to $V_{\text {ref }} / 2$ where $V_{\text {ref }}$ is the power supply voltage of the SAR ADC. This first analog approximated value is obtained in the first cycle. Third, if the first sample is higher than $V_{\text {ref }} / 2$, the MSB value is maintained at " 1 ," unless it becomes " 0 " in the second cycle. In this cycle, still for the same sample, the SARs move to the next bit and initialize it at "1." The SARs operate similarly as for the MSB. Then, they move to the next bit until they reach the least significant bit (LSB).

After $1 / F_{\text {ref }}$ period, the following sample is compared to the first analog approximated value, $V_{\text {ref }} / 2$, approximated and converted as it was explained in the previous paragraph for the first sample. The SAR ADC architecture is presented in Fig. 2.

The SAR ADC is simplified in two basic blocks of sampling and quantization. The $\mathrm{S} / \mathrm{H}$ or reference frequency, $F_{\text {ref }}$, is $1 \mathrm{kHz}$ as it is the commonly used value to sample ECG signals, whereas the resolution, $m$, varies from 8 to 16 as presented in Table 1. To obtain test ECG signals that are representative of analog signals, each ECG data vector, $\mathrm{ECG}_{\text {data }}$,

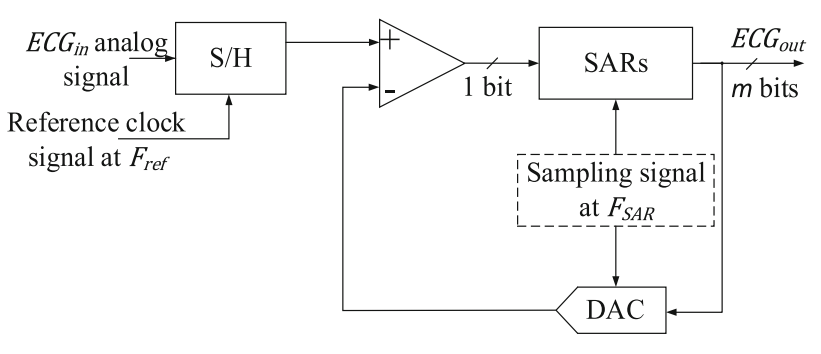

Fig. 2 SAR ADC architecture

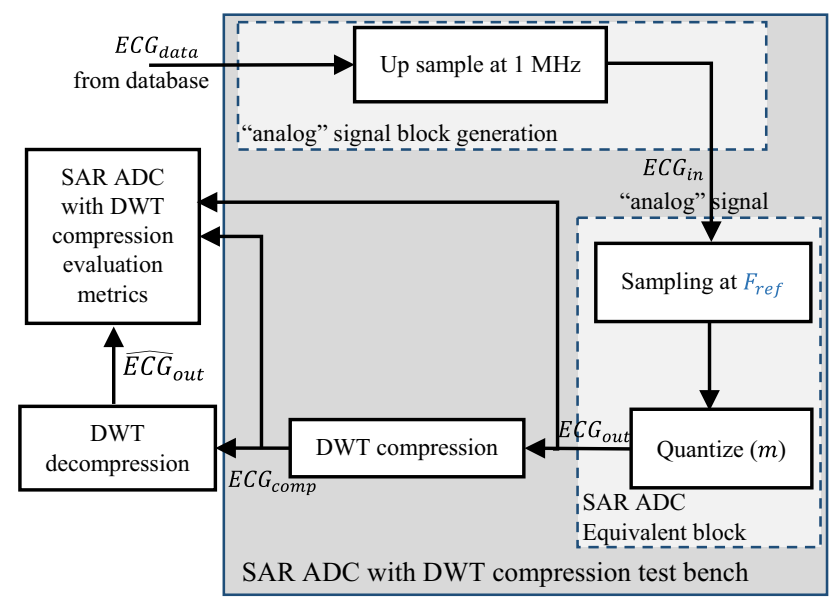

Fig. 3 Performance evaluation test bench of (A1) the SAR ADC with DWT compression

from a given database, is up-sampled at $1 \mathrm{MHz}$ to obtain a signal with a fine time axis. The obtained "analog" signal is sampled at $F_{\text {ref. The }} i$ th analog sample is quantized to the digital sample, $\mathrm{ECG}_{\text {out }}(i)$, using the data length, $m$, that is, the SAR ADC resolution. DWT compression is operated on $L$ ECG digital samples and delivers $L$ compressed data, $\mathrm{ECG}_{\text {comp }}$, which length in bits is $\mathrm{Lb}_{\text {comp. }}$. In this paper, the performance evaluation test bench of (A1) the SAR ADC with DWT compression is illustrated in Fig. 3 in order to test SAR ADC with DWT compression.

To evaluate the quality of the DWT compression regarding the original uniform ECG data, $\mathrm{ECG}_{\text {out }}$, DWT irreversible decompression is applied to $\mathrm{ECG}_{\text {comp }}$ to estimate $\widehat{\mathrm{ECG}}_{\text {out }}$ that is compared to $\mathrm{ECG}_{\mathrm{out}}$. However, before dealing with evaluation metrics, the authors describe the DWT compression methodology in the next subsection.

\subsection{Discrete Wavelet Transform for Compression}

In this subsection, first, a brief mathematical definition of the DWT and its relationship with compression is given as reported in the literature. Second, some wavelet matrices are provided as examples for a better understanding of this paper. Third, the methodology of the compression study is introduced. 
The signal decomposition is generally performed for lossless transforms using orthogonal wavelets or biorthogonal wavelets that are invertible [59]. The transformed signal, $s$, has the same length as the original one and is a representation of the original signal in an orthogonal or invertible matrix. The original signal is perfectly recovered as it requires the transposition of an orthogonal decomposition matrix, or the inversion of an invertible decomposition matrix. These transformations are lossless, whereas they separate the signal from nonsignificant data.

Indeed, the DWT is a set of linear operations performed on an $L$-length vector of sampled signal, $\mathrm{ECG}_{\text {out }}$. It generates a vector of discrete data, $s$, that will be exploited instead of $\mathrm{ECG}_{\text {out }}$ to perform many tasks in signal processing [60]. The matrix form of these linear projections is given in (4) where $\Phi$ is an $L$-by- $L$ decomposition or analysis matrix with $\phi_{i}$ its $i$ th row and $\alpha_{i}$ the $i$ th obtained coefficient. The coefficient, $\alpha_{i}$, results from the scalar product of $\mathrm{ECG}_{\text {out }}$ and $\phi_{i}$. In fact, each coefficient, $\alpha_{i}$, compares the signal $\mathrm{ECG}_{\text {out }}$ to the row $\phi_{i}$.

\section{$\Phi \cdot \mathrm{ECG}_{\mathrm{out}}=s$}

with $\left\langle\phi_{i}, \mathrm{ECG}_{\text {out }}\right\rangle=\alpha_{i}$ and $s=\left\{\alpha_{i}, 1 \leq i \leq L\right\}$

Thus, $s$ is a different representation of $\mathrm{ECG}_{\text {out }}$ in the analysis matrix described by the rows in $\Phi$. The exact recovery of $\mathrm{ECG}_{\text {out }}$ from these coefficients is done by multiplying both left sides of (4) with the inverse matrix of $\Phi$ that is noted $\Psi$. This gives (5) where $\Psi$ is the wavelet or synthesis matrix. For orthogonal matrix, $\Psi$, the columns of $\Psi, \psi_{i}$, are the rows of $\Phi$.

$\mathrm{ECG}_{\text {out }}=\Psi_{S}$

Noting that the DWT compression rounds the small coefficients to zeros, thus invertibility or reversibility is lost and the transformation becomes lossy. Therefore, the number, $K$, is the number of the most significant coefficients which are higher than a given threshold, Th, and is small compared to the length of $s$. The set of the coefficients $\left\{\alpha_{i}>\mathrm{Th}\right\}$ corresponds to the DWT compression output, $E C G_{c o m p}$, as presented in Fig. 3. Its data length is $K$. If $\alpha_{i}$ is higher than Th , the wavelet $\psi_{i}$, which is the $i$ th column in $\Psi$, is active in $\mathrm{ECG}_{\text {out }}$. Furthermore, if $\mathrm{ECG}_{\text {out }}$ is compressible in $\Phi$, the nonsignificant coefficients in $s$ are rounded to zeros. From (5), the recovery of $\mathrm{ECG}_{\text {out }}$ is approximated from its projections as in (6),
$\widehat{E C G}_{\mathrm{out}}=\sum_{u} \alpha_{u} \psi_{u}$

with $u \in\left\{i \mid \alpha_{i}>\mathrm{Th}, 1 \leq i \leq L\right\}$ and $K=\operatorname{card}\{i\}$

where $\widehat{\mathrm{ECG}}_{\text {out }}$ is the decompressed data after the DWT compression of $\mathrm{ECG}_{\mathrm{out}}$ and $K$ is the number of the active columns in $\mathrm{ECG}_{\text {out }}$ regarding $\alpha_{i}>$ Th.

For an orthogonal matrix, $\left\{\phi_{i}, 1 \leq i \leq L\right\}$, the irreversible decompression is done with the $K$ active columns of $\Psi$ that are the rows of $\Phi$. For an orthogonal matrix with no normalized rows to unit, the irreversible decompression is done with the $K$ active columns of $\Psi$ and the matrix $\Phi \Psi$ is diagonal. For a biorthogonal matrix, the rows of $\Phi$ are biorthogonal to the columns of $\Psi$ and the decompression is also irreversible. Thus, each scalar product $\phi_{i} \psi_{j}$ is equal to 1 only if $i$ is equal to $j$ for $1 \leq i \leq L$ and $1 \leq j \leq L$. Besides, both orthogonal and biorthogonal wavelet matrices can be implemented due to tree-structured filter banks. The filter coefficients of the analysis tree are deduced from the decomposition matrix factorization. Meanwhile, the filter coefficients of the synthesis tree are deduced from the wavelet matrix factorization [59]. The number of matrices in a factorization corresponds to $J$ which is the wavelet decomposition level. The filter bank is tree-structured with $J$ low-pass (LP) filters and $J$ high-pass (HP) filters in both analysis and synthesis banks. LP filters in the analysis bank select signal information. These LP filters outputs are known as approximations. However, HP filters in the analysis bank select noise part of the signal. These HP filters outputs are known as details. The approximations and details are truncated to $m$ bits that are the SAR ADC resolution. The selected values that are higher than the threshold, Th, constitutes the DWT compression output, $\mathrm{ECG}_{\text {comp }}$. Its length in bits, $\mathrm{Lb}_{\text {comp }}$, is equal to $m$ times $K$ that is the number of the selected coefficients, $\mathrm{ECG}_{\mathrm{comp}}$. Therefore, in this paper, the DWT compression as a lossy compression is selected for implementation with tree-structured filter banks since the authors study an embedded solution for IoT healthcare devices. The online DWT compression of clinically and nonclinically relevant ECG parts is preferred to off-line solution with lossless compression of clinically relevant ECG parts and lossy compression of nonclinically relevant ECG parts after ECG data segmentation.

The DWT decomposition is generally performed due to the orthogonal wavelets such as Coiflet (coif), Daubechies (db), Symlet (sym), Dmeyer (dmey) and Haar (haar) families, or the biorthogonal wavelets such as biorthogonal (bior) and reverse biorthogonal (rbio) families [61]. For each family, there are one or more subfamilies according to wavelet family parameters that impact numbers and values of the filter coefficients. After the SAR ADC, DWT-based ECG data compression often uses Daubechies or biorthogonal wavelets. These two wavelet examples of orthogonal and 
biorthogonal wavelet families are widely used and yield good compression ratios [53] even though, to quantitatively compare (A1) the SAR ADC with DWT compression to (A2) the LC-ADC, it is necessary to select the best-fit wavelet subfamily. The objective is to select the best DWT compression to be implemented after the SAR ADC considering the compression ratio, the ECG signal distortion and the implementation complexity. Thus, to select the best-fit wavelet subfamily regarding only the compression ratio, BCR, and the ECG signal distortion, $\mathrm{PRD}_{\text {comp }}$, the orthogonal matching pursuit (OMP) algorithm is used to obtain an approximation of the signal in the $L$-by- $L$ dictionary consisting of wavelet subfamily where $L$ corresponds to the number of the SAR ADC samples. In fact, the OMP algorithm aims at computing all coefficients, $\left\{\alpha_{i}, 1 \leq i \leq L\right\}$, and selecting the $K$ most significant ones, based on an iterative application of the DWT [62]. Thus, $K$ is provided as the convergence criterion and is also defined as the minimum number of iterations to guarantee a given $\mathrm{PRD}_{\text {comp }}$ since the OMP selects the most active row of $\Phi$ at each iteration. The methodology of the DWT compression study is defined in order to evaluate DWT compression on a uniform ECG data, $\mathrm{ECG}_{\text {out, }}$, and for a given wavelet subfamily, as shown in Fig. 4. The DWT compression study, organized in 5 steps, is proposed to select the best-fit wavelet subfamily according to the compression ratio and the ECG signal distortion. The proposed study is achieved through MATLAB simulations including the functions of wavelet toolbox, statistics and machine learning toolbox as well as the signal processing toolbox. In fact, the simulations are based on the function of the denoising and compression wavelet family to perform the OMP algorithm. This algorithm is developed for the sake of rapid MATLAB programming and simulation results.

The orthogonal matching pursuit (OMP) algorithm's running time is dominated by the comparison of the data $\mathrm{ECG}_{\text {out }}$ or the residual to the rows $\phi_{i}$ which complexity is $\mathrm{O}\left(K L^{2}\right)$ [63]. First, to reduce this complexity and for given decomposition matrix dimensions, $D \times D, \mathrm{ECG}_{\text {out }}$ is split into $n$ successive segments $\left\{\mathrm{ECGt}_{\mathrm{out}_{l}}, 1 \leq l \leq n\right\}$. Each segment, $\mathrm{ECGt}_{\mathrm{out}_{l}}$, is composed of $D$ successive samples, and $\mathrm{ECG}_{\text {out }}$ is truncated to ECGt $\mathrm{Eut}_{\text {out }}$ The $\mathrm{ECGt}_{\text {out }}$ length, $\hat{L}$, is given in (7), where $L$ is the $E G_{\text {out }}$ data length and $\left.L.\right\rfloor$ is the floor function.

$\hat{L}=n D$ with $n=\left\lfloor\frac{L}{D}\right\rfloor$

Second, the OMP is applied separately to each segment, $\mathrm{ECGt}_{\mathrm{out}_{l}}$, for a given value of the wavelet decomposition level, $J$, and for the maximum number of iterations, $K^{\prime}$. Thus, each segment is compressed to $\mathrm{ECGt}_{\mathrm{comp}_{l}}$ which length is equal to $K^{\prime}$. Third, the original segment is recovered to obtain $\widehat{\mathrm{ECG}}_{\text {out }_{l}}$. In fact, the decomposition matrix is also

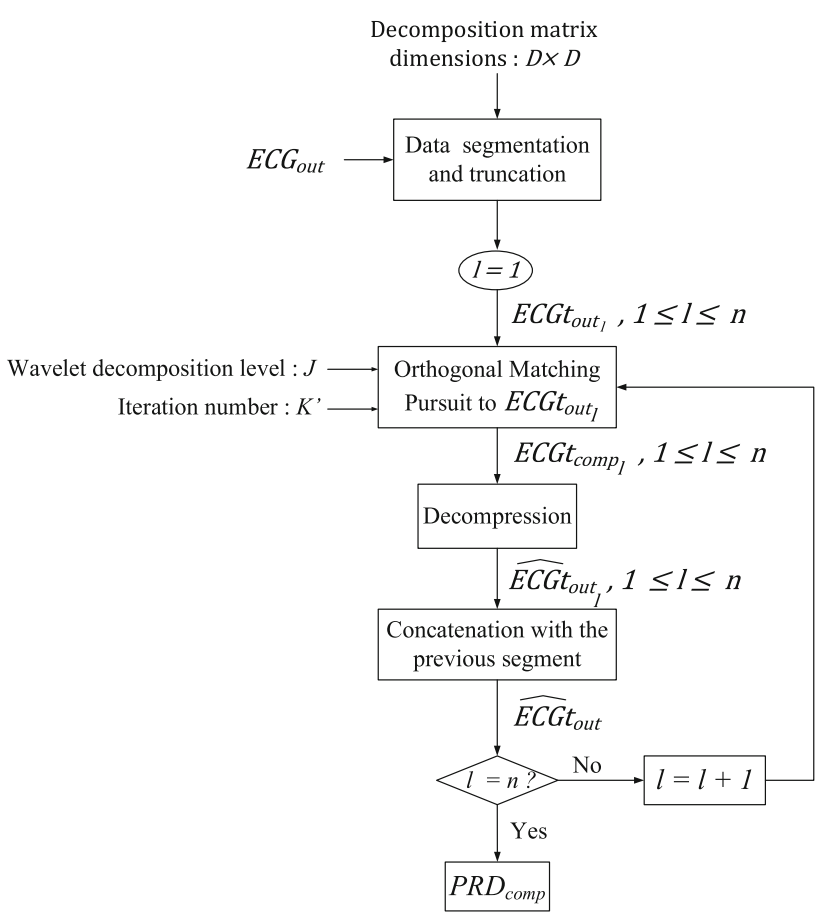

Fig. 4 Flowchart of DWT compression study

applied separately to each $\mathrm{ECGt}_{\mathrm{comp}_{l}}$ using (4) and (6) where $\mathrm{ECG}_{\text {out }}$ is equal to $\mathrm{ECGt}_{\text {out }_{l}}, K$ is equal to $K^{\prime}$ and $L$ is equal to $D$.

Fourth, from the second segment, also equal to the second iteration, $l=2$, of the DWT compression algorithm study given in Fig. 4, the recovered segment, $\widehat{\mathrm{ECG}}_{\mathrm{out}_{l}}$, is concatenated to the previous recovered segment, $\widehat{\mathrm{ECG}}_{\text {out }_{l-1}}$. At the $n^{\text {th }}$ iteration, all the recovered segments are finally concatenated to obtain $\widehat{\mathrm{ECG}}_{\text {out }}$ that represents the recovered data of $\mathrm{ECGt}_{\text {out }}$. Consequently, the OMP complexity becomes $O$ $\left(n K^{\prime} D^{2}\right)$ where $K^{\prime}$ is the number of the selected rows from the decomposition matrix to represent each $D$-length segment. Fifth, the PRD ${ }_{\text {comp }}$ is computed to evaluate the DWT compression in terms of recovered signal quality for a given signal and a given subfamily.

Thus far, the five steps extract $\mathrm{PRD}_{\text {comp }}$ variations versus $K^{\prime}$ for given wavelet subfamily, decomposition matrix dimensions, $D \times D$, and wavelet decomposition level, $J$. It

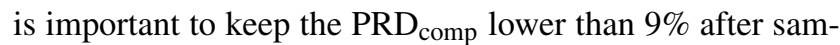
pling, quantizing and compression as it is the criterion for a low distortion and thus for a good diagnosis quality [43]. Due to ECG temporal and amplitude range variations [64], the number of coefficients, $K^{\prime}$, for a $9 \% \mathrm{PRD}_{\text {comp varies from }}$ one signal to another. Therefore, the data compression efficiency evaluation is applied to all wavelet subfamilies for given dimension, $D$, and wavelet decomposition level, $J$, as far as many test ECG signals are concerned. A statistical comparison is processed, and the number of the optimal coefficients, $K_{\text {opt }}^{\prime}$, is obtained for the selected best-fit DWT. Aside 
from compression, transmission then diagnosis purposes as it is in this paper, DWT can also be used in the training process in order to extract significant ECG parameters. These parameters are keys to identify patients [2].

Finally, performances are evaluated after the best-fit wavelet subfamily selection for compression of uniform ECG data. The LC-ADC model is presented, and the performance evaluation test bench is proposed in the next subsection before evaluation metrics definitions to compare them to (A2) the LC-ADC performances.

\subsection{LC-ADC Model and Evaluation}

In this paper, the best candidate of (A2) the LC-ADC to tackle the comparative study to (A1) the SAR ADC with DWT compression is the floating-window type. Indeed, it has been a widely used and tested type during the two last decades [34, $38,65]$. The ECG analog signal, $\mathrm{ECG}_{\mathrm{in}}$, is continuously compared to two reference levels. The in-between difference is the LC-ADC quantization step, $q$, that is given in (8) where FS is the LC-ADC full scale and $M$ is the LC-ADC resolution.

$q=\frac{\mathrm{FS}}{2^{M}}$

The two comparator outputs are INC and DEC regarding the up- or down-crossing direction, respectively. When an event is detected by the comparators, digital values of the reference levels are updated. Then, the digital-to-analog converter (DAC) delivers the updated analog reference levels. These steps result in increment or decrement of both crossed levels by $q$. The floating-window type inherits its name from the simultaneous update of both analog levels. Moreover, a logic control sets the digital value of the crossed level, $\mathrm{ECG}_{\text {outLC, }}$ as the output sample [66]. In fact, the LC-ADC signal dependency is due to this behavior. Thus, when the signal amplitude is not varying, the LC-ADC is not activated. That makes it more suitable for ECG signal acquisition than a Nyquist or sigma-delta ADC that samples ECG signal at each time period. In order to recover the signal from the LCADC samples, $\mathrm{ECG}_{\text {outLC }}$, the time information is needed to time-localize the level crossings (LCs). Therefore, the timetracking stage includes a synchronized counter at a reference counter frequency, $F_{\mathrm{C}}$. This counter enumerates the number of periods, $1 / F_{\mathrm{C}}$, to obtain the time interval, TI, between two successive LCs. The reference counter resolution is equal to $N$ bits.

The block diagram of the described LC-ADC architecture is shown in Fig. 5. A detailed LC-ADC model is already implemented in MATLAB/SIMULINK and designed according to ECG signal specifications in terms of amplitude and frequency [66]. In order to recover the

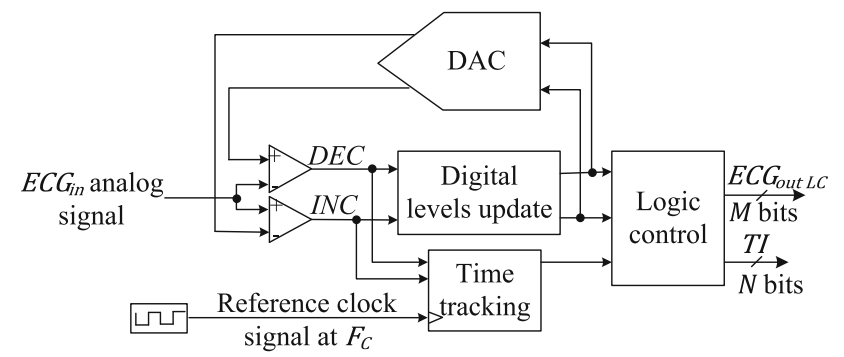

Fig. 5 LC-ADC block diagram

ECG signal, the samples at the LC-ADC output, ECG $_{\text {outLC, }}$, besides the time intervals at the LC-ADC output, TI, are required. Alternatively, only DEC and INC outputs of both comparators instead of all samples, $\mathrm{ECG}_{\text {outLC, }}$ are enough to be transmitted to a distant control unit. In fact, from the first sample, $\mathrm{ECG}_{\text {outLC }}(1)$, DEC values, INC values together with the LC-ADC quantum, the samples, $\mathrm{ECG}_{\text {outLC, }}$ can be computed with digital level updates and the logic control blocks.

Behavioral simulations are done with modeled ECG signals for normal, bradycardia and tachycardia [66] and then tested with real ECG records for normal, emotional or physical duress and various pathologies from Physionet database [21]. In fact, the LC-ADC is designed and modeled to cope with all ECG signals that give heart rates from 30 beats per minute (bpm) to $240 \mathrm{bpm}$ [66]. Thus, the LC-ADC full scale, $\mathrm{FS}$, is set to $10 \mathrm{mV}$, the reference counter frequency, $F_{\mathrm{C}}$, is equal to $10 \mathrm{kHz}$, and the reference counter resolution, $N$, is 12 bits for an $M$-bit LC-ADC where $M$ is equal to 8 . In the present paper, the LC-ADC performance evaluation test bench is illustrated in Fig. 6. As in Fig. 3, to obtain test ECG signals that represent analog signals, the input data, $\mathrm{ECG}_{\text {data }}$, are up-sampled at $1 \mathrm{MHz}$ to obtain the "analog" signal, $\mathrm{ECG}_{\text {in }}$, with a fine time axis. This "analog" signal is the LC-ADC model input. Besides, the signal reconstruction to obtain uniformly sampled signal at $F_{\text {ref }}, \widehat{\mathrm{ECG}}_{\text {outLC }}$, is operated due to linear interpolation with a $p$-bit resolution. In fact, the $1 \mathrm{kHz}$ reconstruction frequency and the $p$-bit resolution are the same as the $\mathrm{S} / \mathrm{H}$ frequency, $F_{\text {ref }}$, and the resolution, $m$, respectively, in the SAR ADC to perform the quantitative comparative study. Afterward, the LC-ADC evaluation metrics are computed from three vectors of data obtained during the same time duration. They are the samples at the LC$\mathrm{ADC}$ output, $\mathrm{ECG}_{\text {outLC, }}$, and the linear interpolation output, $\widehat{\mathrm{ECG}}_{\text {outLC}}$, but also the SAR ADC output after truncation, ECGt $_{\text {out }}$, as described in Fig. 3.

To show comparative curves of LC-ADC output and SAR ADC output, SAR ADC is chosen with 16-bit resolution and $1 \mathrm{kHz}$ reference frequency. The test ECG data are the V1 lead of s0044lre from PTB diagnostic ECG (ptbdb) [21]. An example of samples at the LC-ADC output versus the sam- 


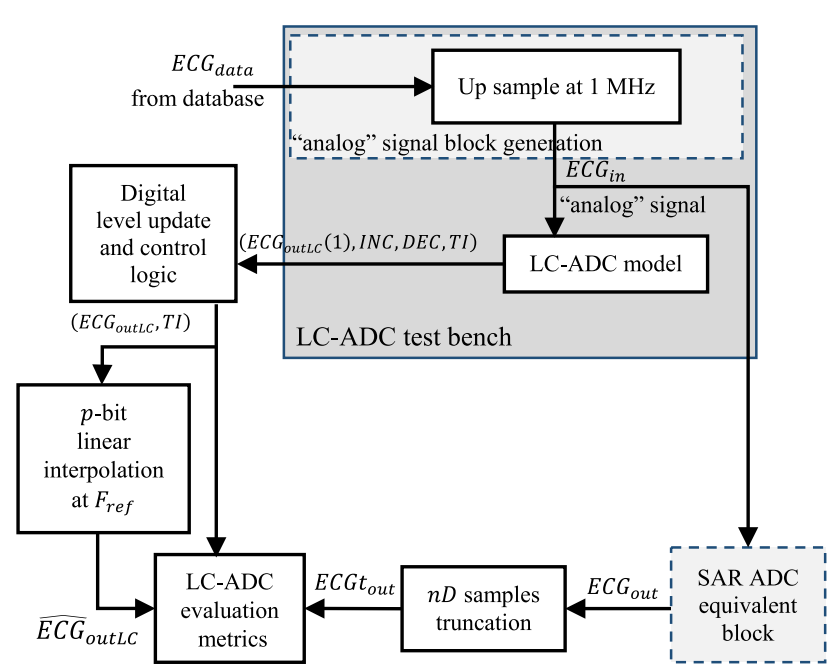

Fig. 6 Performance evaluation test bench of (A2) the LC-ADC

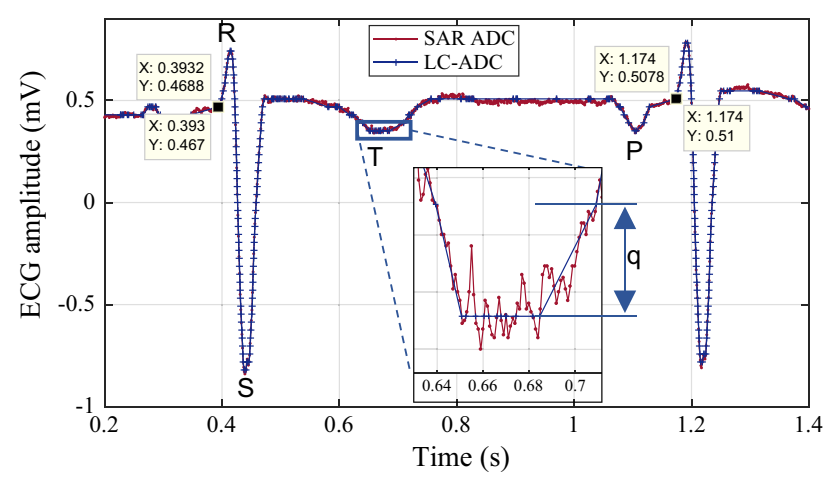

Fig. 7 LC-ADC output versus ECG SAR ADC output

ples at the SAR ADC output is given in Fig. 7. The patient of s0044lre has a mean cardiac rhythm of 67 beats per minute (bpm) and suffers from myocardial infarction which is a challenging medical emergency. In this example, the $\mathrm{Q}$ wave, that is, generally before the $\mathrm{R}$ wave, is not observed. Between the pointed data cursors, the LC-ADC delivers $S$ samples, equal to 184 , whereas the SAR ADC delivers $L$ samples, equal to 782 samples.

Between the pointed data cursors in Fig. 7, the SAR ADC delivers $L$ samples, equal to 782 samples of 16 bits each. Alternatively, the LC-ADC delivers $S$ samples. This number $S$ is equal to 184 which needs 3680 bits if 8 bits per $\mathrm{ECG}_{\text {outLC }}$ and 12 bits per TI are sent. However, the LC-ADC can deliver only INC and DEC that are the required bits aside with the first crossed level, $\mathrm{ECG}_{\text {outLC }}(1)$, to update LC-ADC levels and to provide the exact samples at the LC-ADC output before linear interpolation. This optimization makes the amplitude output requires only 376 bits. As far as the time intervals, TI, are concerned, they require 2208 bits for $S$ samples. Thus, instead of 12512 bits at the SAR ADC output, the LC-ADC can send only 2584 bits. Thus, this LC-ADC with data optimization represents the architecture (A2). In case of (A1) the SAR ADC with DWT compression at BCR equal to $70 \%$, the data length of 12,512 bits becomes equal to 3754 bits. That confirms the SAR ADC output compression's importance of test ECG signals or the use of LC-ADC amplitude output optimization.

For the V1 lead of s0044lre, Fig. 8 shows different values of time, amplitude and total data lengths in order to compare the data lengths at the outputs of (A1) the SAR ADC with DWT compression, (A2) the LC-ADC and other architectures. In fact, six architectures are compared. They are the standalone SAR ADC, (A1) the SAR ADC with DWT compression, the SAR ADC with DWT compression which is followed by Huffman compression, the LC-ADC with complete amplitude output, (A2) the LC-ADC with optimization of amplitude output, the LC-ADC with optimization of amplitude output and Huffman compression which is applied to time intervals. In this paper, (A2) the LC-ADC means that the LC-ADC is tested with optimization of amplitude output. For the LC-ADC with optimization of amplitude output and Huffman compression, the time intervals, TI, could be compressed by a lossless compression method. However, this is not the aim of this paper as Huffman will be a further compression after the DWT compression or of the LC-ADC time interval output. Thus, the authors carry out their comparative study between (A1) and (A2).

This paper focuses on the event-driven ECG sensor to reduce the message data length. The aim is to optimize data transmission from a healthcare device to a distant control unit in order to analyze patients' ECG signals after ECG signal reconstruction, features' extraction can be added, and then, patients' identification and authentication can be operated. The authors detail performance evaluation metrics in the next subsection in order to highlight the advantage of the eventdriven ECG sensor regarding SAR ADC-based ECG sensor. Selected ECG signals, $\mathrm{ECG}_{\mathrm{data}}$, for the inputs of performance evaluation test benches are also included.

\subsection{ECG Signals and Evaluation Metrics}

Performance evaluation test benches of (A1) the SAR ADC with DWT compression and (A2) the LC-ADC are illustrated in Figs. 3 and 6, respectively. The test ECG data at their input, $\mathrm{ECG}_{\text {data }}$, are downloaded from Physionet [21]. In this paper, (A1) and (A2) are tested with 50 different normal or pathological ECG signals. These signals are introduced in Annex A. The selected signals cover normal and apnea, ECG signals in addition to many pathological ECG signals but also ECG signals during emotional or physical duress which is described by ST segment variation. Pathologies are from bradycardia, arrhythmia, atrial fibrillation and tachycardia to the most critical one which is myocardial infarction. The selected signals are from many ECG databases as Apnea 


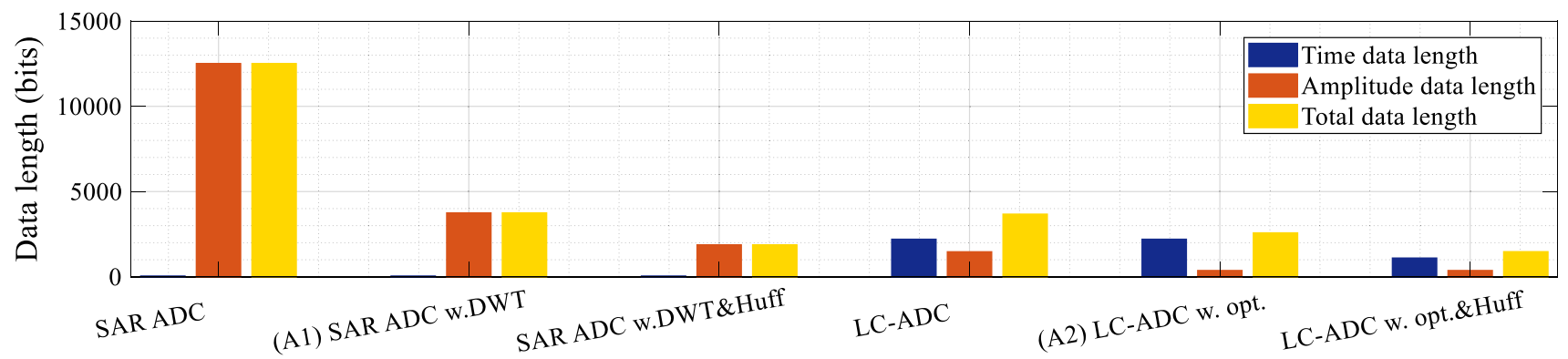

Fig. 8 ECG sensor with different signal acquisition and data compression architectures, namely (A1) the SAR ADC with DWT compression and (A2) the LC-ADC with optimization of data transmission

ECG, MIT-BIH arrhythmia (mitdb), PTB diagnostic ECG (ptbdb), long-term ST (lstdb), MIT-BIH atrial fibrillation (afdb), MGH-MF waveform (mghdb) which have different resolutions and sampling frequencies.

Evaluation metrics of (A1) the SAR ADC with DWT compression and (A2) the LC-ADC performance evaluation test benches are computed during the same given time duration, DT. Evaluation metrics of (A1) the SAR ADC with DWT compression performance evaluation test bench are cited below.

- The DWT compression percent root-mean-square difference, PRD $_{\text {SAR \&DWT }}$, is computed to evaluate the recovered signal quality after DWT decompression.

- The data length of the SAR ADC with DWT compression, data_length $h_{S A R \& D W T}$, is the data length in bits of the selected coefficients after DWT compression during DT.

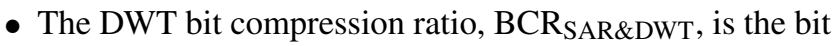
compression ratio of DWT compression which is applied on the SAR ADC output.

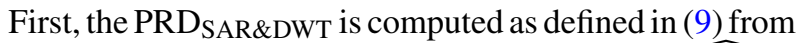
(3) where $L$ is replaced by $n D, \mathrm{ECG}_{\text {out }}$ by $\mathrm{ECGt}_{\text {out }}, \widehat{\mathrm{ECG}}_{\text {out }}$ by $\widehat{\mathrm{ECG}}_{\text {out }}$ and $\overline{\mathrm{ECG}}_{\text {out }}$ by $\overline{\mathrm{ECGt}}_{\text {out }}$.

$$
\begin{aligned}
& \text { PRD }_{\text {SAR\&DWT }}(\%) \\
& =100 \times \sqrt{\frac{\sum_{i=1}^{n D}\left(\mathrm{ECGt}_{\mathrm{out}}(i)-\widehat{\mathrm{ECG}}_{\mathrm{out}}(i)\right)^{2}}{\sum_{i=1}^{n D}\left(\mathrm{ECGt}_{\mathrm{out}}(i)-\overline{\mathrm{ECGt}}_{\mathrm{out}}\right)^{2}}}
\end{aligned}
$$

In fact, the first step of the DWT compression study in Fig. 4 is the segmentation of $L$-length $\mathrm{ECG}_{\text {out }}$ into $n$ segments of length equal to $D$ and their concatenation into $\hat{L}$-length

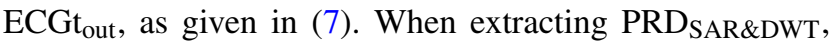
results must be discussed according to SAR ADC effective number of bits (ENOB). Indeed, ENOB represents the real resolution of the ADC. Thus, signal quality of the overall SAR ADC with DWT compression is PRD SAR\&DWT versus ENOB and not versus the SAR ADC resolution, $m$.
Second, the DWT compression is applied $n$ times on $n$ segments, $\mathrm{ECGt}_{\text {outl }}$, which length is equal to $D$. The data length, $n K^{\prime}$, is thus obtained from the coefficients' data length of the DWT compression, ECGt comp $_{\text {. Moreover, as each coefficient }}$ is quantized to $m$ bits, the data length in bits of (A1) is given in (10).

data_length $h_{\mathrm{SAR} \& \mathrm{DWT}}(\mathrm{bits})=m n K^{\prime}$

Conversely, the bit compression ratio, BCR $\mathrm{BAR}_{\mathrm{SAWT}}$, of SAR ADC with DWT compression depends only on the BCR of DWT compression as defined in (2). Indeed, third, BCR $_{\text {SAR\&DWT is computed from (2) by replacing } L \text { by } n D}$ and $\mathrm{Lb}_{\text {comp }}$ by data_length $h_{\text {SAR\&DWT }}$ which is defined in (10). The third evaluation metric of (A1) is thus given in (11).

$\operatorname{BCR}_{\operatorname{SAR} \& D W T}(\%)=\frac{m n D-m n K^{\prime}}{m n D}=\frac{D-K^{\prime}}{D}$

If the operated DWT compression corresponds to the selected best-fit DWT that returns $K_{\mathrm{opt}}^{\prime}$ optimal coefficients, data_length SAR\&DWT in (10) and BCR $_{\text {SAR\&DWT in (11) }}$ have to be computed for $K^{\prime}$ equal to $K_{\mathrm{opt}}^{\prime}$.

In this paper, to compare performances of (A1) the SAR ADC with DWT compression and (A2) the LC-ADC, the SAR ADC number of samples, $n D$, and the LC-ADC number of samples, $S$, must be taken in a given duration, DT, respectively.

Evaluation metrics of (A2) the LC-ADC performance evaluation test bench are four:

- The LC-ADC percent root-mean-square difference, $\mathrm{PRD}_{\mathrm{LC}}$ - ADC, is computed to evaluate the recovered signal quality after linear interpolation.

- The LC-ADC mean sampling frequency, $F_{\text {mean }}$, is computed to approximate the data output frequency.

- The LC-ADC data length, data_length $h_{\mathrm{LC}}$ - ADC, is the data length in bits of the LC-ADC outputs during DT. 
- The LC-ADC bit compression ratio, $\mathrm{BCR}_{\mathrm{LC}}$ - $\mathrm{ADC}$, is computed to evaluate the data reduction at the LC-ADC outputs.

As far as the PRD $\mathrm{LC}_{\mathrm{ADC}}$ is concerned, on the one hand, the ECG signal quality is evaluated after linear interpolation at $F_{\text {ref }}$ to recover $p$-bit uniformly sampled ECG signal, $\widehat{\mathrm{ECG}}_{\text {outLC. }}$ On the other hand, during DT, ECG $\mathrm{EutLC}$ has $S$ samples. Consequently, the recovered digital signal, $\widehat{\mathrm{ECG}}_{\text {outLC }}$, has exactly $n D$ samples during the same duration, DT. The PRD $\mathrm{LC}_{\text {- ADC }}$ is hence defined in (12) where ECGt $_{\text {out }}$ is the SAR ADC equivalent output after truncation to $n D$ samples as given in Fig. 6 .

$$
\begin{aligned}
& \operatorname{PRD}_{\mathrm{LC}-\mathrm{ADC}}(\%) \\
& =100 \times \sqrt{\frac{\sum_{i=1}^{n D}\left(\mathrm{ECGt}_{\mathrm{out}}(i)-\widehat{\mathrm{ECG}}_{\mathrm{outLC}}(i)\right)^{2}}{\sum_{i=1}^{n D}\left(\mathrm{ECGt}_{\mathrm{out}}(i)-\overline{\mathrm{ECGt}}_{\mathrm{out}}\right)^{2}}}
\end{aligned}
$$

However, from the time intervals at the LC-ADC output, the mean sampling frequency, $F_{\text {mean }}$, is computed as in (13) where $\mathrm{TI}_{i}$ is the $i$ th time interval between two successive LCs, $F_{\mathrm{C}}$ is the reference counter frequency and $S$ is the number of time intervals.

$F_{\text {mean }}=\left(\frac{1}{S . F_{\mathrm{C}}} \sum_{i=1}^{S} \mathrm{TI}_{i}\right)^{-1}$

As far as the LC-ADC data length in bits,

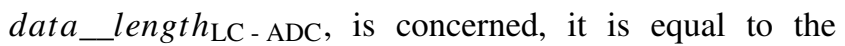
data length in bits of the samples at the LC-ADC output added to the data length in bits of the time intervals at the LC-ADC output. On the one part, during DT, the number of samples, $S$, is reduced as in Fig. 8. In fact, only the first $M$-bit amplitude sample is required besides all the INC and DEC values that describe the other samples, $E C G_{\text {outLC. }}$ Thus, the data length in bits of the samples at the LC-ADC output is equal to $M+2(S-1)$. On the other part, the data length in bits of the time intervals is $N S$ with $N$ the reference counter resolution. Finally, the data_length $h_{\mathrm{LC}-\mathrm{ADC}}$ is given in (14).

data_length LC $-\mathrm{ADC}_{(\mathrm{bits})}=(N+2) S+M-2$

By the end, the LC-ADC bit compression ratio, $\mathrm{BCR}_{\mathrm{LC}}$ - ADC, derives from the definition in (2). Indeed, $L$ is replaced by $n D$ because of truncation of the samples at the SAR ADC output, $m$ by $p$ which is the linear interpolation resolution, and $\mathrm{Lb}_{\mathrm{comp}}$ by data_length $h_{\mathrm{LC}-\mathrm{ADC}}$. Thus, the last evaluation metric of (A2) is given in (15).

$\mathrm{BCR}_{\mathrm{LC}-\mathrm{ADC}}(\%)=\frac{p n D-((N+2) S+(M-2))}{p n D}$
The next section introduces MATLAB simulation results regarding the performance evaluation test benches. Then, it deals with quantitative comparison between (A1) the SAR ADC with DWT compression and (A2) the LC-ADC in terms of the defined evaluation metrics.

\section{Evaluation Results and Discussions}

To conduct the compression study, 50 ECG signals were applied as test input. They are normal and pathologic ECG signals selected with different shapes, amplitude ranges and pathology types from Physionet database [21]. There are also Apnea-ECG signals and ECG signals during emotional or physical duress which is described by ST segment variation. Each ECG input signal, $\mathrm{ECG}_{\text {data }}$, is applied at the input of the evaluation performance test bench of the SAR ADC with DWT compression. The SAR ADC samples at $1 \mathrm{kHz}$ reference frequency. To compress SAR ADC output, all the 54 available MATLAB wavelet subfamilies of the Coiflet (coif), Daubechies (db), Symlet (sym), DMeyer (dmey), biorthogonal (bior) and reverse biorthogonal (rbio) families are generated using MATLAB functions. Simulations are operated considering three parameters: the decomposition matrix dimensions, $D \times D$, the wavelet decomposition level, $J$, and the number of selected coefficients to compress the

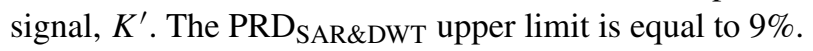

This section starts with some preliminary results and bestfit wavelet subfamily with $K^{\prime}, D$ and $J$ selection for the SAR ADC with DWT compression. Afterward, the performances are discussed according to the selected DWT, $K^{\prime}, D$ and $J$. In addition, each test ECG signal, $\mathrm{ECG}_{\mathrm{data}}$, is also applied at the input of the evaluation performance test bench of the LCADC. Then, the results for the LC-ADC output are presented and discussed. Finally, the authors compare the compression and signal quality of (A1) the SAR ADC with DWT compression versus (A2) the LC-ADC in terms of percent root-mean-square difference, bit compression ratio and data length in bits.

\subsection{Preliminary Results}

Preliminary simulations were done for a single test ECG signal which is the I lead of s0044lre from PTB diagnostic ECG (ptbdb) with all the wavelet subfamilies of MATLAB. Each subfamily is set for wavelet decomposition levels, $J$, from 2 to 4 , and for decomposition matrix dimensions, $D \times D$, equal to $64 \times 64$. Besides, as the decomposition matrix is invertible and matrix dimensions are required to be a power of 2 to design analysis and synthesis filters, the authors observe preliminary results also for $D$ equal to 128,256 and 512 . On the one hand, lower values of $D$ may not show enough decomposition matrix rows with many zeros to obtain data 

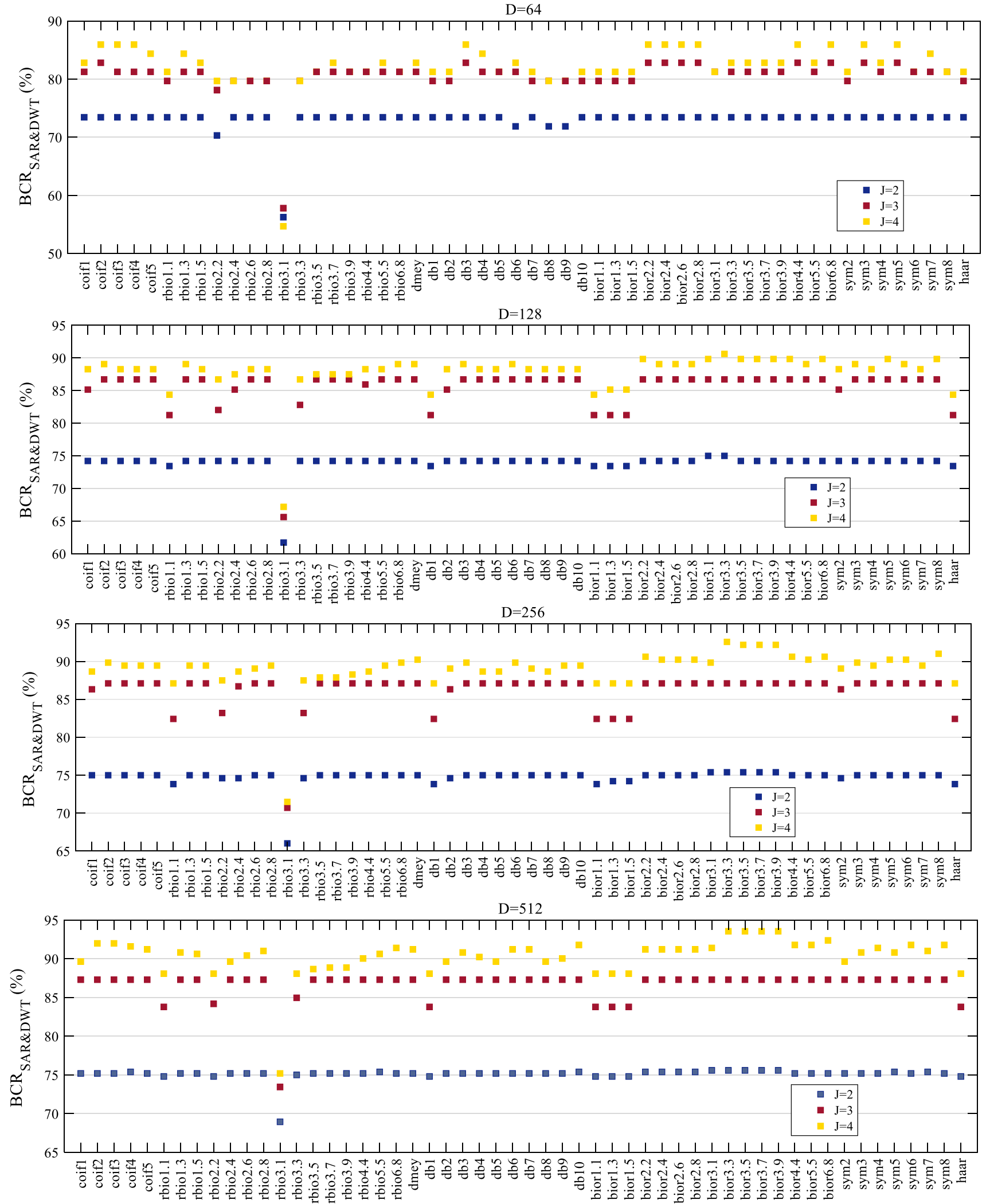

Fig. 9 Bit compression ratio of DWT compression on the SAR ADC output for the I lead of s0044lre ECG signal from PTB diagnostic ECG (ptbdb) for $D=64, D=128, D=256$ and $D=512$ (from top to down) for different values of wavelet decomposition level 
Table 5 Bit compressio ratio, BCR SAR\&DWT $_{\text {( }}$ ), of SAR ADC with DWT compression for bior3.3 and Haar wavelet subfamilies for $D=$ 128

\begin{tabular}{lllll}
\hline DWT & \multicolumn{2}{l}{ Level } & & \\
\cline { 2 - 5 } & 2 & 3 & 4 & 5 \\
\hline bior3.3 & $75 \%$ & $86.72 \%$ & $91.41 \%$ & $92.97 \%$ \\
Haar & $73.44 \%$ & $82.03 \%$ & $85.16 \%$ & $85.94 \%$ \\
\hline
\end{tabular}

decompositions in small and high coefficients. On the other hand, increasing dimensions from 64 to 512 has a slight effect on compression ratio and higher values of $\mathrm{D}$ show precisely the same results. For the selected ECG signal and for a PRDSAR\&DWT upper limit that is equal to $9 \%$, Fig. 9 shows

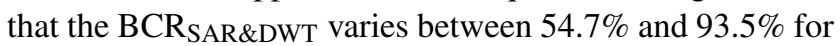
many configurations of $D$ and $J$. For the test ECG signal, the best wavelet subfamilies are the bior3.3, bior3.5, bior3.7 and bior3.9 with $D$ equal to 512 and $J$ equal to 4 . Increasing the wavelet decomposition level, $J$, leads to a better compression performance, namely from 2 to 3 . For example, Table 5

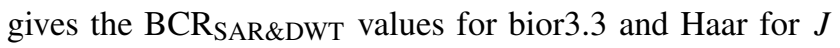
from 2 to 5 . These help to observe that $J$ is fixed till third wavelet decomposition level because for higher values of $J$, performances do not vary significantly. In fact, for bior3.3

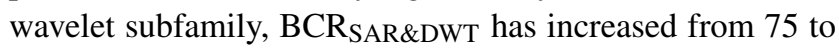
$86.72 \%$ when $J$ varies from 2 to 3 , whereas increasing $J$ from

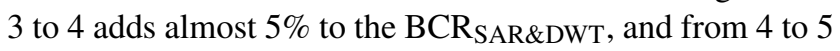
adds only $1.56 \%$ to the previous BCR SAR\&DWT $_{\text {. }}$

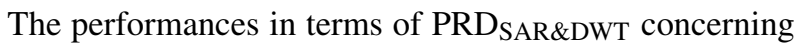
the number of decomposition matrix rows, $K^{\prime}$, retained by the OMP are also analyzed for $(J, D)$ equal to $(2,64),(2$, $128),(3,64)$ or $(3,128)$ for all test ECG signals. For the sake of clarity, these results are not presented in this paper since that requires four figures for each subfamily. As expected, the PRD ${ }_{\text {SAR\&DWT }}$ increases when the number of selected rows, $K^{\prime}$, decreases. In fact, the more decomposition matrix rows are discarded from data representation, the more information is lost. In the case of bior3.3 wavelet subfamily, $18.75-28.12 \%$ of the decomposition matrix rows are needed for a $9 \%$ PRD SAR\&DWT for all the test ECG signals depending on the decomposition matrix dimensions and the wavelet decomposition level. Thus, the correspondent BCR SAR\&DWT varies from 71.87 to $81.25 \%$. The maximum value of $K^{\prime}$ is equal to 17 and 36 for $J$ equal to 2 in the case of 64 and 128 matrix dimensions, respectively. The minimum value of

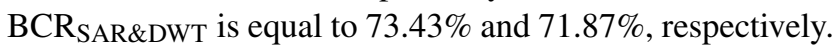
For $J$ equal to 2 and $D$ equal to 64 , at least $26.56 \%$ of bior3.3 wavelet coefficients must not be zeroed to recover ECG signals with $9 \%$ PRD $_{\text {SAR\&DWT }}$ or less. This corresponds to a minimum value of BCR $\mathrm{SAR}_{\text {\&DWT }}$ equal to $73.43 \%$. Furthermore, for $J$ equal to 3 , the minimum value of $K^{\prime}$ is equal to 8 and 11 for $D$ equal to 64 and 128, respectively. These

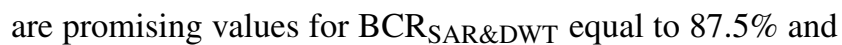
$91.4 \%$ but guarantee a $9 \%$ PRD $_{\text {SAR\&DWT }}$ only for one test ECG signal. To sum up, preliminary simulation results show that bior3.3 wavelet subfamily is not always the best choice to compress the uniformly sampled ECG signals if the $9 \%$ PRD ${ }_{\text {SAR\&DWT }}$ criterion is respected. The best-fit DWT selection results are presented in the next subsection.

\subsection{Best-Fit DWT Selection}

To achieve a complete study of the DWT compression after SAR ADC, all available MATLAB orthogonal and biorthogonal subfamilies have been applied to the 50 real ECG signals as in the performance evaluation test bench of Fig. 3. Compression results are obtained for $(L, D)$ equal to $(2,64),(2$, $128),(3,64)$ or $(3,128)$. Using 54 wavelet subfamilies, compression results are studied with MATLAB boxplot function from statistics and machine learning toolbox. In fact, the boxplot function is a statistical representation that describes the data distribution by delimiting with a box its 25 th percentile (first quartile) and 75th percentile (third quartile), and by two horizontal lines its minimum and maximum values. It also indicates with a line inside the box the median value of the distributed data.

In Fig. 10, a statistical variation range of $B_{C A} R_{\text {AR\&DWT }}$ is presented for the wavelet subfamilies. Using the flowchart in Fig. 4, the number of the most significant coefficients, $K^{\prime}$, satisfying the $9 \%$ PRD $_{\text {SAR\&DWT varies according to the wavelet }}$ subfamily, $J, D$ and the test ECG signal. Many wavelet subfamilies are not appropriate to the compression of the uniformly sampled ECG signals regarding others, namely the rbio3.1 wavelet. In fact, it presents the highest variation range of $K^{\prime}$ for all decomposition matrix dimensions and wavelet decomposition levels. This choice delivers the lowest variation range of BCR $_{\text {SAR\&DWT }}$ that was already detected in the preliminary results presented in Fig. 9. In Fig. 10 (a), there are some exceptions, known as outliners, that are the values outside the boxplot range between the minimum and maximum values. The outliners are present for subfamilies which median, first quartile and third quartile are identical. Moreover, the range between the maximum and the minimum is proportional to the range between the first and third quartiles. That hence explains the presence of outliners suggesting that some test ECG signals use more or less coefficients than almost all the test ECG signals. For example, db1 selects 17 coefficients and BCR $\mathrm{SAR}_{\mathrm{SDWT}}$ is equal to $73.43 \%$ for almost all the test ECG signals. However, it rarely selects 13,15 or 16 coefficients to obtain higher bit compression ratios.

Still, from Fig. 10, regarding all the subfamilies, the authors focus on the highest value of the first quartile among the 54 subfamilies rather than the highest value of the third quartile, the maximum, the minimum or the median. This ensures that at least $25 \%$ of the test ECG signals satisfy a 
(a) $\mathrm{J}=2$ and $\mathrm{D}=64$

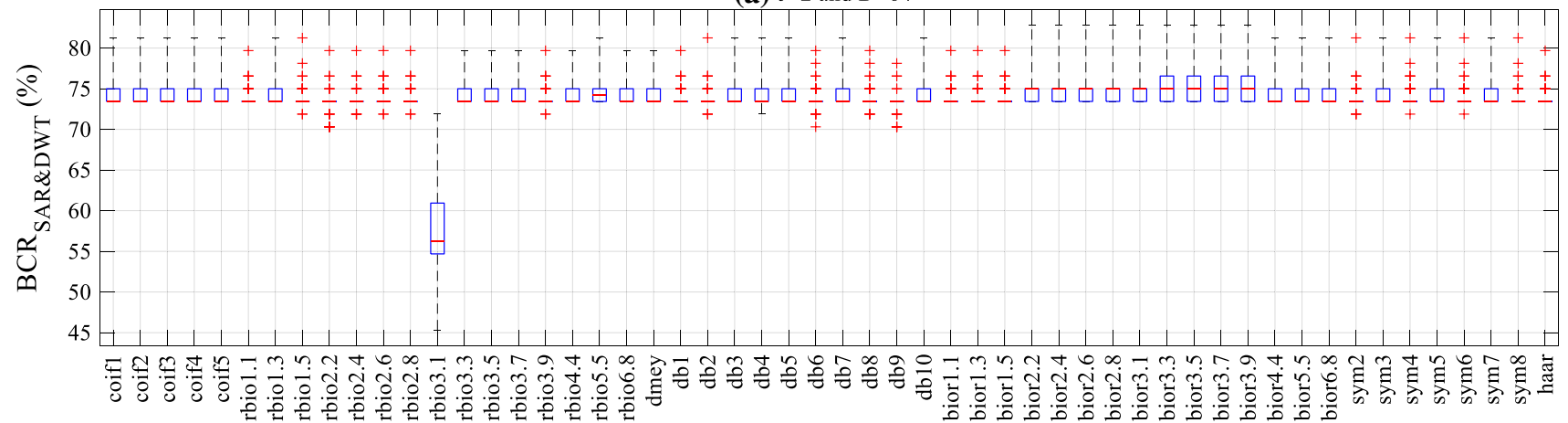

(b) $\mathrm{J}=2$ and $\mathrm{D}=128$

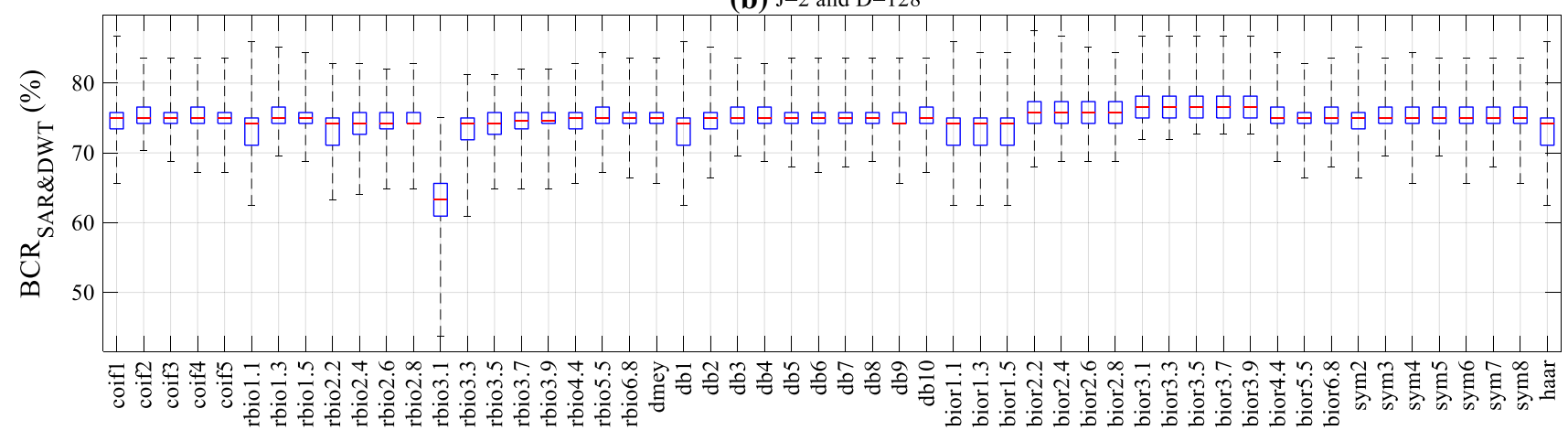

(c) $\mathrm{J}=3$ and $\mathrm{D}=64$
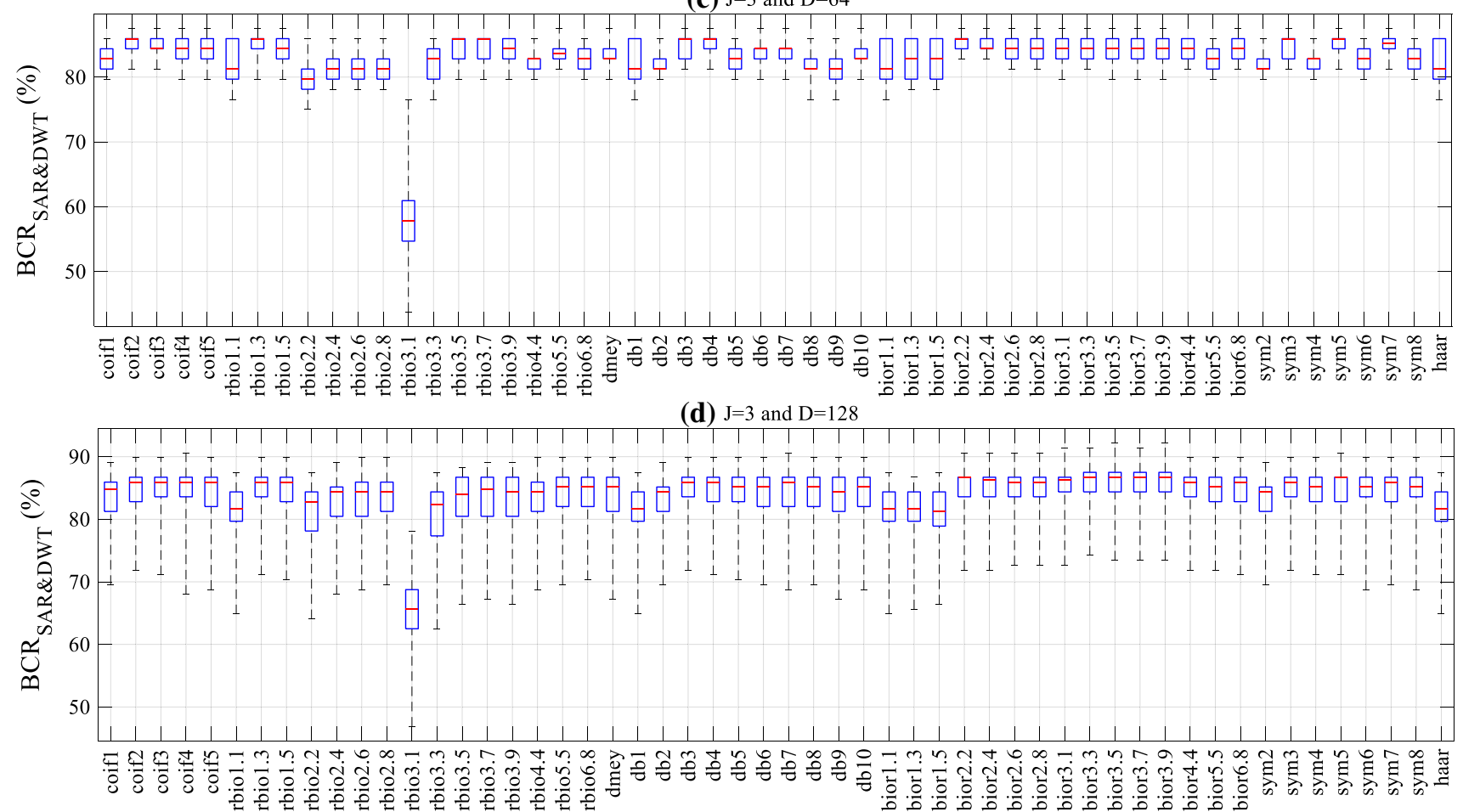

Fig. 10 Selected coefficient number after DWT compression of SAR ADC outputs regarding a PRD equal to 9\% versus wavelet subfamilies for (2, $64),(2,128),(3,64)$ and $(3,128)$ configurations (from top to down)

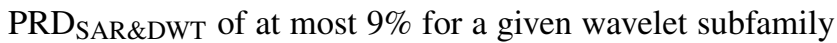

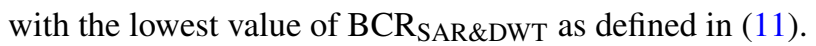

Thus, according to Fig. 10, for $J$ equal to 2 and $D$ equal to 64 , bior3.3, bior3.5, bior3.7 and bior3.9 wavelet subfamilies that ensure $K_{\mathrm{opt}}^{\prime}$ equal to 17 and BCR $\mathrm{SAR}_{\mathrm{S}}$ DW equal to 
Table 6 Wavelet decomposition results for a 9\% PRDSAR\&DWT

\begin{tabular}{|c|c|c|c|}
\hline$\overline{(J, D)}$ & $K_{\mathrm{opt}}^{\prime}$ & BCR $_{\text {SAR\&DWT }}(\%)$ & Wavelet subfamilies \\
\hline$(2,64)$ & 17 & 73.43 & $\begin{array}{l}\text { bior } 3.3 \text {, bior } 3.5, \\
\text { bior3.7, bior3.9 }\end{array}$ \\
\hline$(2,128)$ & 32 & 75.00 & $\begin{array}{l}\text { bior } 3.1, \text { bior3 } 3 \text {, } \\
\text { bior3.5, bior3.7, } \\
\text { bior3. } 9\end{array}$ \\
\hline$(3,64)$ & 10 & 84.37 & $\begin{array}{l}\text { coif } 2, \text { rbio1.3, db4, } \\
\text { bior } 2.2, \text { sym } 5\end{array}$ \\
\hline$(3,128)$ & 20 & 84.37 & $\begin{array}{l}\text { bior3.3, bior3.5, } \\
\text { bior3.7, bior } 3.9\end{array}$ \\
\hline
\end{tabular}

$73.43 \%$ are the selected ones. These subfamilies can reach a $76.56 \%$ BCR $_{\text {SAR \&DWT }}$ for $75 \%$ of the test ECG signals. These results and those for other configurations of $J$ and $D$ are summarized in Table 6.

In Table 6, it is shown that selected subfamilies are similar except for $(J, D)$ equal to $(3,64)$. Besides, dimension increase does not importantly affect the compression ratio but requires two times larger number of ECG samples to be compressed. For $D$ equal to 64 and wavelet decomposition level, $J$, going from 2 to 3 increases 1.5 times the complexity of the analysis tree. In fact, three high-pass (HP) filters and three low-pass (LP) filters are required instead of two HP filters and two LP filters. For example, for bior3.3 subfamily which is the least complex among selected subfamilies for $(J, D)$ equal to $(2,64)$, each LP analysis filter has 8 coefficients and each HP analysis filter has 4 coefficients. However, in the biorthogonal family, bior2.2 and bior3.1 subfamilies have the least numbers of filter coefficients. The factorized values of filter coefficients are given in Table 7 . The number of operations to evaluate filter coefficients is the same, but the bior 2.2 filter processing requires more processing cycles as it needs 5 cycles instead of 4 for bior3.1. In addition, from an implementation point of view, using those biorthogonal wavelet subfamilies leads to implement multiplier-free filters in the tree-structured filter banks. As the coefficients of such filters are the addition of powers of 2 , there is no added quantization error while performing DWT decomposition. The quantization is only used to code $\sqrt{2}$ on $m$ bits which is equal to the SAR ADC resolution.

Thereby, following the SAR ADC and for all test ECG signals, the best-fit configuration of DWT compression is obtained for bior3.1 subfamily with $J$ equal to $2, D$ equal to $64, K^{\prime}$ equal to 17 and thus BCR SAR\&DWT equal to

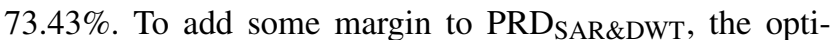
mal value of $K^{\prime}, K_{\mathrm{opt}}^{\prime}$, is fixed at 18 . Thus, BCR $\mathrm{BAR}_{\mathrm{S}} \& W T$ is always equal to $71.875 \%$ as the chosen value of 18 coefficients out of 64 coefficients is obtained from 64 samples at the SAR ADC output regardless SAR ADC resolution. The SAR ADC with DWT compression performance eval-
Table 7 Bior 2.2 and bior3.1 analysis filter coefficients

\begin{tabular}{ll}
\hline & Filter coefficients \\
\hline $\begin{array}{l}\text { bior2.2 } \\
\text { LP analysis filter }\end{array}$ & $\sqrt{2} \times\left[0,-2^{-3}, 2^{-2}, 2^{-2}+2^{-1}, 2^{-2},-2^{-3}\right]$ \\
bior2.2 & $\sqrt{2} \times\left[0,2^{-2},-2^{-1}, 2^{-2}, 0,0\right]$ \\
HP analysis filter & $\sqrt{2} \times\left[-2^{-2}, 2^{-2}+2^{-1}, 2^{-2}+2^{-1},-2^{-2}\right]$ \\
bior3.1 & $\sqrt{2} \times\left[-2^{-3}, 2^{-3}+2^{-2},-2^{-3}-2^{-2}, 2^{-3}\right]$ \\
LP analysis filter & \\
bior3.1 & \\
HP analysis filter &
\end{tabular}

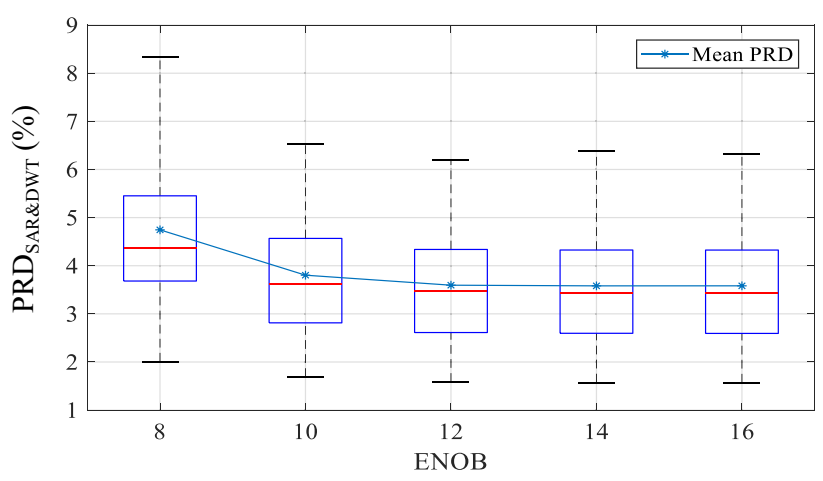

Fig. 11 PRDSAR\&DWT versus SAR ADC effective number of bits where SAR ADC is followed by bior3.1 compression with BCR SAR\&DWT equal to $71.87 \%$

uation test bench of Fig. 3 is then processed for different $\mathrm{ECG}_{\text {data }}$ and configurations. In fact, the test ECG signal

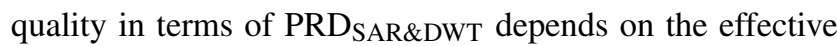
number of bits, ENOB, equal to 8, 10, 12, 14 and 16. The results are summarized in Fig. 11 and show the best values of DWT compression only for ENOB higher than 8. In fact, for

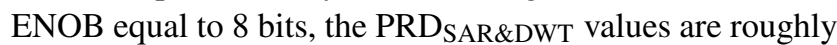
between $3.75 \%$ and $5.5 \%$ for $75 \%$ of the test ECG signals. For ENOB equal to $10,12,14$ and 16, PRD $_{\text {SAR\&DWT }}$ is generally between $2.5 \%$ and $4.5 \%$ for $75 \%$ of the test ECG signals. In the next subsection, the authors compare LC-ADC evaluation metrics, $\mathrm{PRD}_{\mathrm{LC}}$ - ADC,$F_{\text {mean }}$ and $\mathrm{BCR}_{\mathrm{LC}}$ - ADC, for the 50 test ECG signals.

\subsection{LC-ADC Performances}

LC-ADC behavioral simulations are done with the same 50 real ECG records that are used for the SAR ADC with DWT compression performance evaluation test-bench. As explained in Fig. 6, the signals are up-sampled at $1 \mathrm{MHz}$ frequency. This step is done to obtain signals that are representative of analog signals with a fine time axis. Then, since the LC-ADC resolution, $M$, has already been chosen in [66], the converter model is only simulated with a resolution equal to 8 . This value is typical for ECG amplitude quantization and also ensures good quality signal reconstruction which 
Fig. 12 Mean sampling frequency and $\mathrm{BCR}_{\mathrm{LC}}$ - ADC versus ECG signal number with

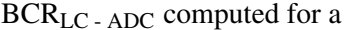
16-bit reconstruction resolution

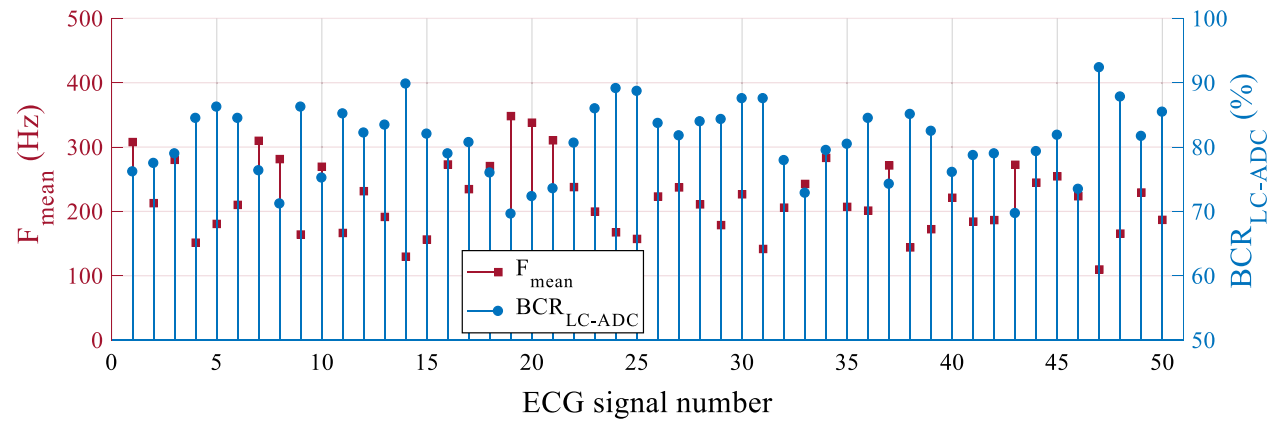

Fig. 13 PRD $D_{L C}$ - ADC and BCR $_{L C}$ - ADC versus ECG signal number for LC-ADC with $\mathrm{BCR}_{\mathrm{LC}}$ - ADC computed for a 16-bit reconstruction resolution

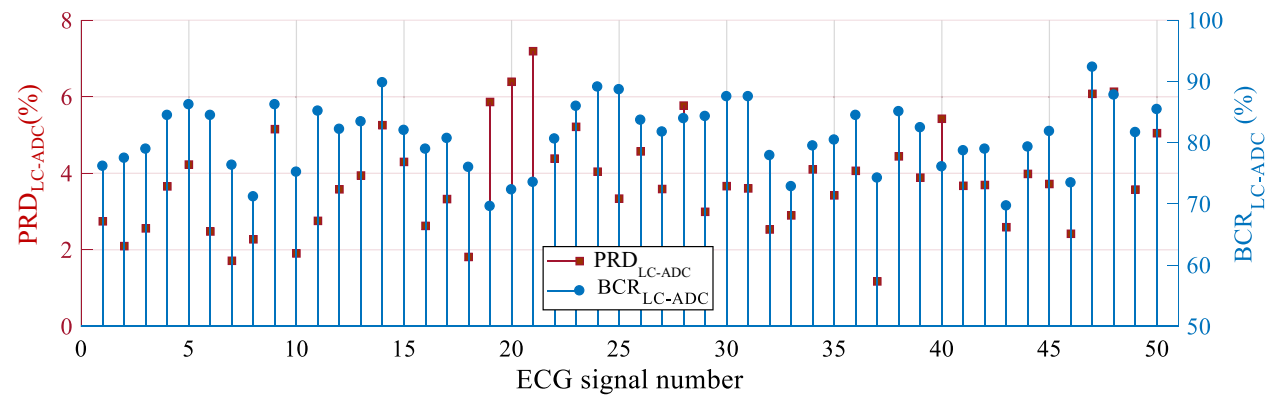

requires at least 8-bit resolution $[67,68]$. The LC-ADC full scale is set to $10 \mathrm{mV}$. As far as the counter is concerned, the resolution, $N$, is 12 bits and the reference counter frequency, $F_{\mathrm{C}}$, is $10 \mathrm{kHz}$ for the 8-bit LC-ADC. For the LC-ADC performance evaluation test bench, simulations are carried out over the same time duration DT of the SAR ADC with DWT compression. The simulations are done during DT equal to one second for every test ECG signal which contains at least one $\mathrm{P}$ wave, one QRS complex and one $\mathrm{T}$ wave.

The $\mathrm{BCR}_{\mathrm{LC}}$ - ADC and the $F_{\text {mean }}$ performances are given in Fig. 12, for all the 50 test ECG signals. Generally, the more the samples at the LC-ADC output are, the more the mean sampling frequency is, the less the bit compression ratio is. The $\mathrm{BCR}_{\mathrm{LC}}$ - $\mathrm{ADC}$ varies from 69.9 to $92.35 \%$. Certainly, and at the opposite of the samples at the SAR ADC output, the results are different from a test ECG signal to another since the number of samples depends on the test ECG signal amplitude and frequency. The 8-bit LC-ADC with 12bit reference counter samples at a mean sampling frequency between $109 \mathrm{~Hz}$ and $348 \mathrm{~Hz}$, whereas generally used SAR ADC sampling frequency is always $1 \mathrm{kHz}$ as provided in the present paper and many works in the literature.

As far as the PRD $D_{L C}$ - ADC values are concerned, they are also evaluated for all test ECG signals and presented

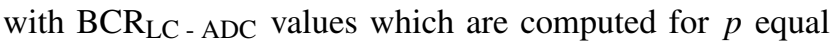
to 16 bits in linear interpolation algorithm. The results in Fig. 13 show no correlation between these two performances. The PRD $\mathrm{LC}_{\mathrm{LC}}$ ADC values vary from 1.25 to $7.25 \%$, whereas $\mathrm{BCR}_{\mathrm{LC}-\mathrm{ADC}}$ values move from 69.9 to $92.35 \%$. To sum up, LC-ADC sampling efficiency depends on the test ECG sig- nal. In fact, for high-frequency and high-amplitude waves, the LC-ADC activity increases.

The next subsection compares the percent root-meansquare difference, the bit compression ratio and the data length in bits of (A1) the SAR ADC with DWT compression and (A2) the LC-ADC in ECG sensors.

\subsection{LC-ADC Versus SAR ADC with bior3.1 Compression}

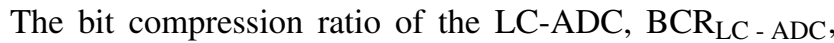
as given in (15), is computed for a $p$-bit reconstruction resolution and $1 \mathrm{kHz}$ reconstruction frequency. Indeed, the LC-ADC input is an analog signal, the LC-ADC output is a digital compressed data, whereas compression ratio is a digital performance. For a given signal, the $\mathrm{BCR}_{\mathrm{LC} \text { - } \mathrm{ADC}}$ values increase when reconstruction resolution, $p$, varies from $8,10,12,14$ to 16 . For instance, if $p$ is equal to 16 , the reconstruction recovers a 16-bit code every $1 \mathrm{~ms}$ independently from their positions in the test ECG signal. Thus, for a given signal, it is obvious that the 16-bit reconstructed LC-ADC output is more performant in terms of bit compression ratio than lower reconstruction resolutions. As far as (A1) is concerned, DWT compresses all the SAR ADC samples including clinically and nonclinically relevant parts in the test ECG signal. Therefore, as shown in Fig. 14, the BCR $_{\text {SAR\&DWT }}$ is equal to $71.87 \%$ for all the test ECG signals. Adversely, LC-ADC compression is signal dependent. More than $50 \%$ of the test ECG signals are compressed with

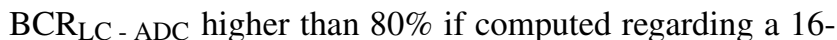
bit reconstruction resolution. For $p$ equal to $8,75 \%$ of test 


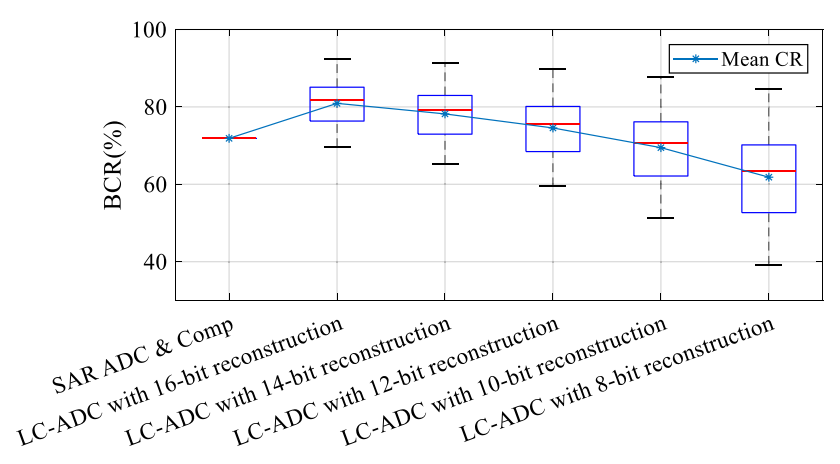

Fig. 14 BCR $_{\text {SAR\&DWT }}$ for SAR ADC with best-fit DWT compression versus $\mathrm{BCR}_{\mathrm{LC}}$ - $\mathrm{ADC}$ where the $\mathrm{BCR}_{\mathrm{LC}}$ - $\mathrm{ADC}$ is computed regarding $p$ bit reconstruction resolution

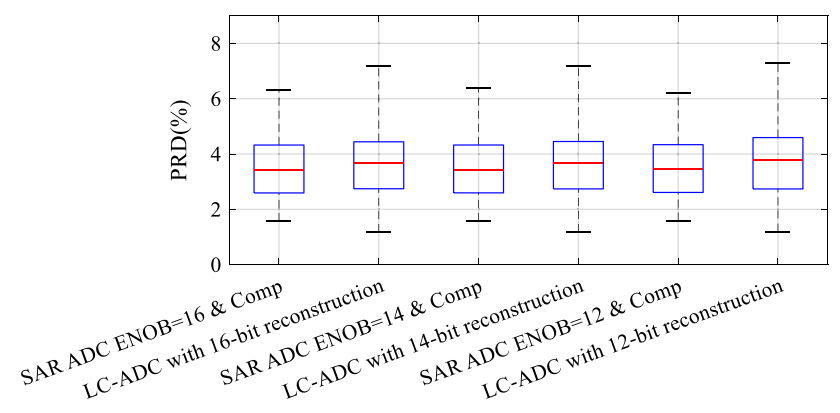

Fig. 15 PRD $_{\text {SAR } \& W T}$ for SAR ADC of ENOB equal to 12, 14 or 16 with best-fit DWT compression versus PRD $\mathrm{LC}_{\text {- ADC }}$ which is computed for $p=\mathrm{ENOB}$

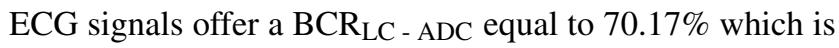
almost the same value as BCR SAR\&DWT for the best-fit DWT compression with $J$ equal to 2 and $D$ equal to 64 after $1-\mathrm{kHz}$ SAR ADC.

As mentioned in the SAR ADC state of the art, a 10-bit SAR ADC provides an ENOB of 6.63 and a 12-bit SAR ADC provides an ENOB of 11.77. The authors in this subsection are interested only in ENOBs that are equal to 12, 14 and 16. Thus, PRD results are summarized in Fig. 15 where the PRDSAR\&DWT results are computed for SAR ADC with the best-fit DWT compression. That is compared to the

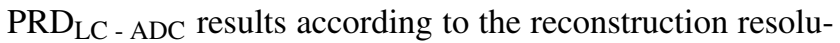
tions, $p$, which are equal to the selected values of ENOB. In fact, the LC-ADC delivers non-uniform samples and requires linear interpolation to recover $p$-bit $1 \mathrm{kHz}$ uniformly sampled signal. The recovered signal is used to evaluate the $\mathrm{PRD}_{\mathrm{LC}}$ - ADC regarding the SAR ADC output for a given value of ENOB equal to $p$. Figure 15 shows that the LCADC almost performs the same as the SAR ADC with DWT compression in terms of PRD. Thus, as this paper aims at optimizing data transmission in order to save data transmission power in RF sensor networks, the authors quantitatively compare data lengths in bits of (A1) the SAR ADC with DWT compression to the ones of (A2) the LC-ADC.

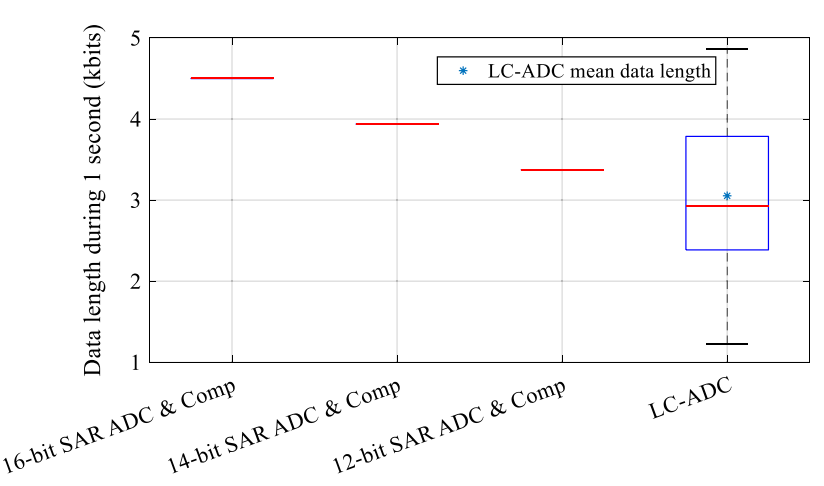

Fig. 16 Data length for $m$-bit SAR ADC with best-fit DWT compression versus LC-ADC data length during one second

Since LC-ADC has non-uniform outputs, the authors need to adopt a same time duration, DT, to compare data length of (A1) to (A2) for all the test ECG signals. If this DT is equal to one second, an $m$-bit SAR ADC at $1 \mathrm{kHz}$ S/H frequency delivers $m$ kbits during one second of conversion. This amount is reduced to $m\left(1-\mathrm{BCR}_{\mathrm{SAR} \& \mathrm{DWT}}\right)$ kbits which is due to the reached compression ratio, BCR $_{\text {SAR\&DWT, equal }}$ to $71.87 \%$. For a 12-bit SAR ADC with DWT compression, this amount is 3.375 kbits. As far as the proposed 8-bit LCADC with 12-bit reference counter is concerned, the mean sampling frequency is between $109 \mathrm{~Hz}$ and $348 \mathrm{~Hz}$ which depends on the test ECG signal.

Besides, it delivers $3.786 \mathrm{kbits}$ for $75 \%$ of test ECG signals during one second of continuous-time sampling as presented in Fig. 16. This data length in bits is located between data lengths at the outputs of 12-bit and 14-bit SAR ADCs with DWT compression. Furthermore, the proposed LC-ADC is more advantageous than a 16-bit SAR ADC with DWT compression for $94 \%$ of the test ECG signals. Thus, it will be more beneficial to use the LC-ADC instead of the SAR ADC with DWT compression especially for recent ECG acquisition systems that require 16-bit resolution [7, 9].

From this quantitative comparison, the performances of (A1) the SAR ADC with DWT compression show similarities, advantages and disadvantages when compared to (A2) the LC-ADC. In fact, they recover approximately the same ranges of percent root-mean-square differences, PRD $D_{\text {SAR\&DWT }}$, as the ones of PRD $\mathrm{LC}_{\text {- ADC }}$, for different values of ENOB and of linear interpolation resolutions. As far as compression ratio is concerned, (A1) the SAR ADC with DWT compression has a constant value of bit compression ratio,and thus, data length is expected to be reduced before data transmission. However, the LC-ADC and 8-bit linear interpolation deliver a $\mathrm{BCR}_{\mathrm{LC}-\mathrm{ADC}}$ equal to $70.17 \%$, for $75 \%$ of the test ECG signals, which is slightly lower than the constant value of compression in (A1). Finally, the drawback of (A1) is that the data length is always constant as the bit compression ratio is constant too. That is considered as 
a drawback because the data length in bits of (A2) is lower than the data length in bits of:

- the 16-bit SAR ADC with DWT compression for $94 \%$ of the test ECG signals,

- the 14-bit SAR ADC with DWT compression for $82 \%$ of the test ECG signals,

- the 12-bit SAR ADC with DWT compression for $68 \%$ of the test ECG signals.

Consequently, if existent SAR ADC-based ECG sensor requires more than 14-bit resolution, the LC-ADC can be perceived as a better architecture to obtain the same signal quality but with lower message data length to transmit to the physician distant control unit. Nonetheless, if the existent SAR ADC-based ECG sensor requires a resolution which is lower than or equal to 12 bits, the ECG sensor's production should be maintained if it meets the patient and the physician requirements.

Finally, the authors conducted this quantitative comparative study while considering the ECG signal quality in the presence of noise and the ECG signal acquisition circuit complexity. On the one hand, the authors have already conducted another work in order to assess the proposed design according to systematic time-quantization error and analog components' non-idealities [66]. The findings of this work can be summarized as follows: First, with offset voltage errors, the PRD $\mathrm{LC}_{\text {- ADC }}$ is kept lower than 9\%, thus reflecting good quality signals. Second, when referring to simulation results with DACs nonlinearities and commercialized DACs specifications, good signal quality was reached.

On the other hand, architecture complexity can be identified according to three performances: circuit area, circuit propagation delay and circuit power consumption. For instance, in $180 \mathrm{~nm}$ CMOS technology, the circuit areas of (A1) and (A2) are approximately equal to $0.45 \mathrm{~mm}^{2}$ and $0.2 \mathrm{~mm}^{2}$, respectively. In fact, SAR ADC area is computed from the mixed-signal chip of [14], DWT compression area is computed from the signal processing chip of [53], and estimated LC-ADC area from the layout is already computed in one of the authors' previous work [66]. Thus, in terms of area the LC-ADC is also a promising architecture. As far as the propagation delay is concerned, the authors evaluate the propagation delays of the comparator which is equal to $5 \mathrm{~ns}$ and the digital levels' update circuit which is equal to $0.8 \mathrm{~ns}$. Added to that, the DAC's settling time in the $180 \mathrm{~nm}$ UMC CMOS technology is estimated to be at most $62 \mathrm{~ns}$. The total delay is at most $68 \mathrm{~ns}$ for the LC-ADC. That is in fact lower than the reference counter period equal to $0.1 \mathrm{~ms}$ to prevent from the increase in the time offset and hence from harmonic distortion. The LC-ADC requires in its architecture an 8-bit DAC that provides the output $62 \mathrm{~ns}$ after the input is injected. Nonetheless, the SAR ADC requires in its architec- ture a 12-bit DAC that provides the output more than $62 \mathrm{~ns}$ after the input is injected. Moreover, the DWT compression will also require more propagation delay to deliver the DWT compression output as it is composed of filters, decimators, detection, estimation and level thresholding [53]. Noting that power consumption is equal to $78.8 \mu \mathrm{W}$ in case of SAR ADC with two different sampling frequencies and Huffman compression for test ECG signal [14], whereas it is only equal to $2.42 \mu \mathrm{W}$ in case of LC-ADC for test sinewave signal [20] which is not really concluding. In fact, to the best of the authors' knowledge, no other works have been conducted to measure power consumption with test ECG signals.

To conclude, PRD of (A1) the SAR ADC with DWT compression and (A2) the LC-ADC are similar despite the presence of noise as in [66]. Distinctively, data length to be transmitted after (A2) is lower than the one to be transmitted after (A1) with 14-bit resolution as shown in Fig. 16 for $82 \%$ of the test ECG signals. In addition, (A2) is also preferable than (A1) covering area, propagation delay and power consumption. Thus, the leaders in design and manufacturing of mixed-signal processing integrated circuits should more investigate on the event-driven ECG sensors. For example, an autonomous wireless sensor node with asynchronous ECG monitoring has been proposed with a $3.8-\mathrm{mm}^{2}$ area and 9.7$\mu \mathrm{W}$ power consumption in $180 \mathrm{~nm}$ CMOS technology [20] but not completely tested with ECG signals yet.

\section{Conclusion}

This paper quantitatively compares the SAR ADC with DWT compression to the LC-ADC starting with ECG signal acquisition and data compression state of the art. Then, it reviews the required tools to deal with the quantitative comparative study, namely the SAR ADC architecture, the DWT definition, its parameters and its related compression method. It also explains the performance evaluation test bench of (A1) the SAR ADC with DWT compression architecture. Moreover, (A2) the LC-ADC architecture, the LC-ADC model and performance evaluation test bench are described as well as the evaluation metrics of both compared architectures. Finally, performance evaluation results are discussed to show similarities between the two architectures in terms of signal quality for different configurations. However, the aim is to optimize data transmission and therefore reduce the ECG sensor power consumption of the RF transmission. Accordingly, the proposed 8-bit LC-ADC with 12-bit and $10-\mathrm{kHz}$ reference counter delivers less data than the 14-bit SAR ADC with DWT compression for $82 \%$ of the test ECG signals. Consequently, the 16-bit or 14-bit SAR ADC would be replaced by the LC-ADC in the commercialized ECG sensors.

This quantitative comparative study and its uses are limited to models, algorithms and simulations with test ECG 
signals from Physionet databases. Meanwhile, SAR ADC results should be improved by the acquisition of ECG signals from existent ECG test devices instead of test ECG signals from Physionet databases. Then, DWT compression can be implemented on field programmable gate array (FPGA) and tested on the acquisition of ECG signals. Besides, more digital signal processing and lossless compression will be also implemented and tested. In fact, a lossless compression, such as the Huffman coding, can be adopted for the DWT selected coefficients. As far as the LC-ADC is concerned, the authors have to send to the foundry the CMOS circuit design files. An LC-ADC test platform will be achieved in order to test ECG signal acquisition. Afterward, the comparison of (A1) the SAR ADC with DWT compression to (A2) the LC-ADC will be more effective. Following that, a lossless compression, such as the Huffman coding, can be adopted after the LC-ADC to the values that are higher than a given amplitude level and to all time intervals. Future works will also deal with an event-driven ECG device with online ECG features' extraction from the LC-ADC optimized outputs, identification and authentication of patients. Additionally to the LC-ADC, the bradycardia requires the highest value of the reference counter resolution that is equal to 12 bits. Meanwhile, other pathologies require lower values of the reference counter resolution. Consequently, pathologycentric event-driven ECG sensors which are different in terms of LC-ADC's reference counter resolution would be proposed.

Acknowledgements The authors would like to thank the CMCU (Comité Mixte de Coopération Universitaire) as it financially supported the Wiobio'ACT project. This project involves cooperation between University of Bordeaux and the Higher School of Communications of Tunis (SUP'COM) from 2015 to 2017. The funding program is Partenariat Hubert Curien-Utique (PHC-Utique) $2015 \mathrm{~N}^{\circ} 32755$ YC.

\section{Annex A}

Test ECG data, $\mathrm{ECG}_{\text {data }}$, are applied at the input of evaluation performances test benches of Figs. 3 and 6. That data are downloaded from Physionet [21], and 50 different normal, apnea or pathological ECG signals are used as well as ECG signals in the presence of emotional and physical duress. The selected signals, as presented in Table 8, cover normal and Apnea-ECG signals in addition to many pathological ECG signals. Pathologies are from bradycardia, arrhythmia, atrial fibrillation and tachycardia to the most critical one which is myocardial infarction.

The selected signals are from many ECG databases as Apnea-ECG (apnea-ecg), MIT-BIH arrhythmia (mitdb), PTB diagnostic ECG (ptbdb), long-term ST (lstdb), MIT-BIH atrial fibrillation (afdb), MGH-MF waveform (mghdb) which have different resolutions and sampling frequencies. A record can contain 1 lead as record "a03" from "apnea-ecg," 2 leads as for record "04048" from "afdb," 12 leads as for " 202 " from "mitdb" and 15 leads as for "s01011re" from "ptbdb." Thus, Table 8 contains information covering record reference and its lead reference, pathology or record description or normal ECG, time duration of the tested segment that contains at least one $\mathrm{P}$ wave, one QRS complex and one $\mathrm{T}$ wave, and sampling information according to the database acquisition. These information are provided to all the test ECG signals that are numbered from 1 to 50. Notations are explained as follows:

(a) Only one available ECG lead

(b) Standard 12-lead ECG

(c) Frank vectorcardiographic system $(\mathrm{Vx}, \mathrm{Vy}, \mathrm{Vz})$

(d) Premature ventricular contractions (PVC)

(e) Two ECG leads that are obtained by ambulatory records

(f) 24-hour ECG holter monitoring with rhythm changes and ECG signal changes

(g) Only two available ECG leads

(h) Atrial premature complexes (APC) 
Table 8 Test ECG signals from Physionet database

\begin{tabular}{|c|c|c|c|c|}
\hline ECG signal number & Record/lead & Pathology or description or normal & Segment duration (s) & Resolution/sampling frequency \\
\hline \multicolumn{5}{|c|}{ Physionet database: Apnea-ECG (apnea-ecg) } \\
\hline 1 & $a 03 / \mathrm{ECG}^{\mathrm{a}}$ & Apnea & 2.57 & $12 \mathrm{bits} / 100 \mathrm{~Hz}$ \\
\hline 2 & $a 05 / \mathrm{ECG}^{\mathrm{a}}$ & & & \\
\hline 3 & $a 07 / \mathrm{ECG}^{\mathrm{a}}$ & & & \\
\hline 4 & $a 10 / \mathrm{ECG}^{\mathrm{a}}$ & & & \\
\hline 5 & $a 11 / \mathrm{ECG}^{\mathrm{a}}$ & & & \\
\hline 6 & $a 15 / \mathrm{ECG}^{\mathrm{a}}$ & & & \\
\hline 7 & $a 16 / \mathrm{ECG}^{\mathrm{a}}$ & & & \\
\hline 8 & $a 17 / \mathrm{ECG}^{\mathrm{a}}$ & & & \\
\hline \multicolumn{5}{|c|}{ Physionet database: MIT-BIH arrhythmia (mitdb) } \\
\hline 9 & $202 / \mathrm{II}^{\mathrm{b}}$ & Bradycardia (49-59 bpm) & 2.85 & $11 \mathrm{bits} / 360 \mathrm{~Hz}$ \\
\hline 10 & $217 / \mathrm{II}^{\mathrm{b}}$ & $\mathrm{PVC}^{\mathrm{a}}$ & & \\
\hline 11 & $217 / \mathrm{V} 1^{\mathrm{b}}$ & & & \\
\hline 12 & $219 / \mathrm{II}^{\mathrm{b}}$ & Bradycardia (38-59 bpm) & & \\
\hline 13 & $221 / \mathrm{V} 1^{\mathrm{b}}$ & Atrial fibrillation (47-110 bpm) & & \\
\hline \multicolumn{5}{|l|}{14} \\
\hline 15 & $223 / I^{\mathrm{b}}$ & $\mathrm{PVC}^{\mathrm{a}}$ & & \\
\hline 16 & $230 / \mathrm{II}^{\mathrm{b}}$ & $\begin{array}{l}\text { Wolff-Parkinson-White (WPW) } \\
\text { syndrome }\end{array}$ & & \\
\hline 17 & $231 / \mathrm{II}^{\mathrm{b}}$ & Second-degree heart block & & \\
\hline 18 & $233 / \mathrm{II}^{\mathrm{b}}$ & $\mathrm{PVC}^{\mathrm{a}}$ & & \\
\hline \multicolumn{5}{|c|}{ Physionet database: PTB diagnostic ECG (ptbdb) } \\
\hline 19 & s0044lre/I $\mathrm{I}^{\mathrm{b}}$ & Myocardial infarction & 2.05 & 16 bits/1000 Hz \\
\hline 20 & s0044lre/II ${ }^{\mathrm{b}}$ & & & \\
\hline 21 & s0044lre/aVR ${ }^{\mathrm{b}}$ & & & \\
\hline 22 & s0044lre/V1 ${ }^{\mathrm{b}}$ & & & \\
\hline 23 & s0044lre/aVL ${ }^{\mathrm{b}}$ & & & \\
\hline 24 & s0101lre/V2 ${ }^{\mathrm{b}}$ & & & \\
\hline 25 & s0101lre/V3 ${ }^{\mathrm{b}}$ & & & \\
\hline 26 & s0101lre/V5 $5^{\mathrm{b}}$ & & & \\
\hline 27 & s0101lre/V6 ${ }^{\mathrm{b}}$ & & & \\
\hline 28 & s0101lre/ $/ \mathrm{x}^{\mathrm{c}}$ & & & \\
\hline \multicolumn{5}{|c|}{ Physionet database: long-term ST (lstdb) } \\
\hline 29 & $s 20011 / \mathrm{II}^{\mathrm{e}}$ & ST segment variation & 4.1 & $12 \mathrm{bits} / 250 \mathrm{~Hz}$ \\
\hline 30 & $s 20011 / \mathrm{V} 2^{\mathrm{e}}$ & & 2.05 & \\
\hline 31 & $s 20041 / \mathrm{ECG}^{\mathrm{f}}$ & & 1.02 & \\
\hline 32 & $s 20111 / \mathrm{V} 2^{\mathrm{e}}$ & & 4.1 & \\
\hline 33 & $s 20141 / \mathrm{II}^{\mathrm{e}}$ & & 2.05 & \\
\hline \multicolumn{5}{|c|}{ Physionet database: MIT-BIH atrial fibrillation (afdb) } \\
\hline 34 & 04048/ECG1 $1^{\mathrm{g}}$ & Atrial fibrillation & 2.05 & $12 \mathrm{bits} / 250 \mathrm{~Hz}$ \\
\hline 35 & 04746/ECG2 ${ }^{\mathrm{g}}$ & & & \\
\hline 36 & 04936/ECG2 ${ }^{\mathrm{g}}$ & & & \\
\hline 37 & $05121 / \mathrm{ECG}^{\mathrm{g}}$ & & & \\
\hline
\end{tabular}


Table 8 continued

\begin{tabular}{|c|c|c|c|c|}
\hline ECG signal number & Record/lead & Pathology or description or normal & Segment duration (s) & Resolution/sampling frequency \\
\hline \multicolumn{5}{|c|}{ Physionet database: PTB diagnostic ECG (ptbdb) } \\
\hline 38 & s0476_re/V2 $2^{\mathrm{b}}$ & Normal & 2.05 & 16bits/1000 Hz \\
\hline 39 & s0476_re/V3 ${ }^{\mathrm{b}}$ & & & \\
\hline 40 & s0476_re/V4 ${ }^{\mathrm{b}}$ & & & \\
\hline 41 & $s 0476 \_r e / \mathrm{Vx}^{\mathrm{c}}$ & & & \\
\hline 42 & s0476_re/V6 $6^{\mathrm{b}}$ & & & \\
\hline 43 & s0476_re/V5 ${ }^{\mathrm{b}}$ & & & \\
\hline \multicolumn{5}{|c|}{ Physionet database: MGH-MF waveform (mghdb) } \\
\hline 44 & $m g h 010 / \mathrm{I}^{\mathrm{b}}$ & Ventricular hypertrophy & 1.42 & $12 \mathrm{bits} / 360 \mathrm{~Hz}$ \\
\hline 45 & $m g h 017 / I^{\mathrm{b}}$ & & & \\
\hline 46 & $m g h 027 / I^{\mathrm{b}}$ & & & \\
\hline \multicolumn{5}{|l|}{47} \\
\hline \multicolumn{5}{|c|}{ Physionet database: MIT-BIH arrhythmia (mitdb) } \\
\hline 48 & $102 / 5^{\mathrm{b}}$ & Paced rhythm & 2.85 & $11 \mathrm{bits} / 360 \mathrm{~Hz}$ \\
\hline 49 & $103 / \mathrm{II}^{\mathrm{b}}$ & APC & & \\
\hline 50 & $103 / \mathrm{V} 2^{\mathrm{b}}$ & APC & & \\
\hline
\end{tabular}

\section{References}

1. Majumder, S.; Chen, L.; Marinov, O.; Chen, C.-H.; Mondal, T.; Deen, M.J.: Non-contact wearable wireless ECG systems for long term monitoring. IEEE Rev. Biomed. Eng. 11(5), 306-321 (2018)

2. Lim, C.L.P.; Woo, W.L.; Dlay, S.S.; Gao, B.: Heartrate-dependent heartwave biometric identification with thresholding-based GMMHMM methodology. IEEE Trans. Industr. Inf. 15(1), 45-53 (2019)

3. Lim, C.L.P.; Woo, W.L.; Dlay, S.S.; Wu, D.; Gao, B.: Deep multiview heartwave authentication. IEEE Trans. Industr. Inf. 15(2), 777-786 (2019)

4. Benjamin, E.J.; Muntner, P.; Alonso, A.; Bittencourt, M.S.; Callaway, C.W.; Carson, A.P.; Chamberlain, A.M.; Chang, A.R.; Cheng, S.; Das, S.R.; Delling, F.N.; Djousse, L.; Elkind, J.F.F.; Fornage, M.; Jordan, L.C.; Khan, S.S.; Kissela, B.M.; Knutson, K.L.; Kwan, T.W.; Lackland, D.T.; Lewis, T.T.; Lichtman, J.H.; Longenecker, C.T.; Loop, M.S.; Lutsey, P.L.; Martin, S.S.; Matsushita, K.; Moran, A.E.; Mussolino, M.E.; O’Flaherty, M.; Pandey, A.; Perak, A.M.; Rosamond, W.D.; Roth, G.A.; Sampson, U.K.A.; Satou, G.M.; Schroeder, E.B.; Shah, S.H.; Spartano, N.L.; Stokes, S.; Tirschwell, D.L.; Tsao, C.W.; Turakhia, M.P.; VanWagner, L.B.; Wilkins, J.T.; Wong, S.S.; Virani, S.S.: AHA statistical update, Heart disease and stroke statistics-2019 update: a report from the American Heart Association. Circulation 139(10), 56-528 (2019)

5. Habte, T.T.; Saleh, H.; Mohammad, B.; Ismail, M.: Ultra Low Power ECG Processing System for IoT Devices. Springer, Basel (2019)

6. Rana, K.P.S.; Kumar, V.; Singhal, A.; Chandel, A.; Pahuja, D.; Vashisht, A.: Time-varying pole-radius IIR multi-notch filters with improved performance. Arab. J. Sci. Eng. 44(8), 7101-7120 (2019)

7. QARDIOCORE the world's first wearable ECG free from patches and wires (CARDIO). http://www.getqardio.com/qardiocorewearable-ecg-ekg-monitor-iphone. Accessed 30 April 2019 (2019)

8. Apple Watch Series 4 proactive health monitor (Apple). http://www.apple.com/uk/apple-watch-series-4/health. Accessed 30 April 2019 (2019)

9. KardiaMobile personal EKG (AlivCor), http://store.alivecor.com/ products/kardiamobile. Accessed 30 April 2019 (2019)
10. Mittal, N.; Singh, U.; Singh Sohi, B.: An energy-aware clusterbased stable protocol for wireless sensor networks. Neural Comput. Appl. 31(11), 7269-7286 (2019)

11. Dong, J.; Jiang, H.; Yang, K.; Weng, Z.; Li, F.; Wei, J.; Ning, Y.; Chen, X.; Wang, Z.: A wireless body sound sensor with a dedicated compact chipset. Circuits Syst. Signal Proc. 36(6), 2341-2359 (2017)

12. Wang, F.; Ma, Q.; Liu, W.; Chang, S.; Wang, H.; He, J.; Huang, Q.: A novel ECG signal compression method using spindle convolutional auto-encoder. Comput. Methods Programs Biomed. 175, 139-150 (2019)

13. Yazicioglu, R.F.; Kim, S.; Torfs, T.; Kim, H.; Hoof, C.V.: A $30 \mu \mathrm{W}$ analog signal processor ASIC for portable biopotential signal monitoring. IEEE J. Solid-State Circuits 460(1), 209-223 (2011)

14. Chen, S.-L.; Villaverde, J.F.; Lee, H.-Y.; Chung, D.W.-Y.; Lin, T.L.; Tseng, C.-H.; Lo, K.-A.: A power-efficient mixed-signal smart ADC design with adaptive resolution and variable sampling rate for low-power applications. IEEE Sens. J. 17(11), 3461-3469 (2017)

15. Gonzalez, R.; Perez, R.; Lopez, M.; Fernandez, I.; Espinosa, J.; Badias, L.; Fernandez, A.; Pena, Y.; Rodriguez, G.: A new tool for heart disease prognosis in the community. Comput. Cardiol. 38, 773-776 (2011)

16. Rijnbeek, P.R.; Kors, J.A.; Witsenburg, M.: Minimum bandwidth requirements for recording of pediatric electrocardiograms. Circulation 104(25), 3087-3090 (2001)

17. Murmann, B.: ADC Performance Survey 1997-2019. http://web. stanford.edu/ murmann/adcsurvey.html. Accessed 11 Feb 2020

18. Rodriguez-Vazquez, A.; Medeiro, F.; Janssens, E.: CMOS Telecom Data Converters. Springer, New York (2003)

19. Kaur, A.; Agarwal, A.; Agarwal, R.; Kumar, S.: A novel approach to ECG R-peak detection. Arab. J. Sci. Eng. 44(8), 6679-6691 (2018)

20. Mansano, A.L.; Li, Y.; Bagga, S.; Serdijn, W.A.: An autonomous wireless sensor node with asynchronous ECG monitoring in $0.18 \mu \mathrm{m}$ CMOS. IEEE Trans. Biomed. Circuits Syst. 10(3), 602-611 (2016) 
21. Moody, G.B.; Mark, R.G.; Goldberger, A.L.: PhysioNet: a webbased resource for the study of physiologic signals. IEEE Eng. Med. Biol. Mag. 20(3), 70-75 (2001)

22. Khan, M.G.: Rapid ECG Interpretation. Springer, New York (2008)

23. Wang, T.-Y.; Li, H.-Y.; Ma, Z.-Y.; Huang, Y.-J.; Peng, S.-Y.: A bypass-switching SAR ADC with a dynamic proximity comparator for biomedical applications. IEEE J. Solid State Circuits 53(6), 1743-1754 (2018)

24. Rahiminejad, E.; Saberi, M.; Lotfi, R.: A power-efficient signalspecific ADC for sensor-interface applications. IEEE Trans. Circuits Syst. II Express Briefs 64(9), 1032-1036 (2017)

25. Yan, L.; Harpe, P.; Pamula, V.R.; Osawa, M.; Harada, Y.; Tamiya, K.; Van Hoof, C.; Yazicioglu, R.F.: A 680 nA ECG acquisition IC for leadless pacemaker applications. IEEE Trans. Biomed. Circuits Syst. 8(6), 779-786 (2014)

26. Sharma, A.; Polley, A.; Lee, S.B.; Narayanan, S.; Li, W.; Sculley, T.; Ramaswamy, S.: A Sub-60- $\mu$ A multimodal smart biosensing SoC with > 80-dB SNR, 35 $\mu$ A photoplethysmography signal chain. IEEE J. Solid State Circuits 52(4), 1021-1033 (2017)

27. Hirai, Y.; Matsuoka, T.; Tani, S.; Isami, S.; Tatsumi, K.; Ueda, M.; Kamata, T.: A biomedical sensor system with stochastic A/D conversion and error correction by machine learning. IEEE Access 7, 21990-22001 (2019)

28. Zhang, Z.; Li, J.; Zhang, Q.; Wu, K.; Ning, N.; Yu, Q.: A dynamic tracking algorithm based SAR ADC in bio-related applications. IEEE Access 6, 62166-62173 (2018)

29. Adimulam, M.K.; Srinivas, M.B.: A 1.0 V, 9.84 fJ/c-s FOM reconfigurable hybrid SAR-sigma delta ADC for signal processing applications. Analog Integr. Circ. Sig. Process 99(2), 261-276 (2019)

30. Chen, C.; Chen, L.; Wang, X.; Zhang, F.: A 0.6 V, $8.4 \mu$ W AFE circuit for biomedical signal recording. Microelectron. J. 75, 105-112 (2018)

31. Mark, J.W.; Todd, T.D.: A nonuniform sampling approach to data compression. IEEE Trans. Commun. 29(1), 24-32 (1981)

32. Kozmin, K.; Johansson, J.; Delsing, J.: Level-crossing ADC performance evaluation toward ultrasound application. IEEE Trans. Circuits Syst. I Regul. Pap. 56(8), 1708-1719 (2009)

33. Li, Y.; Zhao, D.; Serdijn, W.: A sub-microwatt asynchronous levelcrossing ADC for biomedical applications. IEEE Trans. Biomed. Circuits Syst. 7(2), 149-157 (2013)

34. Allier, E.; Sicard, G.; Fesquet, L.; Renaudin, M.: Asynchronous level crossing analog to digital converters. Measurement 37(4), 296-309 (2005)

35. Weltin-Wu, C.; Tsividis, Y.: An event-driven clockless levelcrossing ADC with signal-dependent adaptive resolution. IEEE J. Solid State Circuits 48(9), 2180-2190 (2013)

36. Tang, W.; Osman, A.; Kim, D.; Goldstein, B.; Huang, C.; Martini, B.; Pieribone, V.A.; Culurciello, E.: Continuous time level crossing sampling ADC for bio-potential recording systems. IEEE Trans. Circuits Syst. I Regul. Pap. 60(6), 1407-1418 (2013)

37. Rovere, G.; Fateh, S.; Benini, L.: A $2.2 \mu \mathrm{W}$ cognitive always-on wake-up circuit for event-driven duty-cycling of IoT sensor nodes. IEEE J. Emerg. Sel. Top. Circuits Syst. 8(3), 543-554 (2018)

38. Trakimas, M.; Sonkusale, S.R.: An adaptive resolution asynchronous ADC architecture for data compression in energy constrained sensing applications. IEEE Trans. Circuits Syst. I Regul. Pap. 58(5), 921-934 (2011)

39. Zhang, X.; Lian, Y.: A 300-mV 220-nW Event-driven ADC with real-time QRS detection for wearable ECG sensors. IEEE Trans. Biomed. Circuits Syst. 8(6), 834-843 (2014)

40. Hou, Y.; Qu, J.; Tian, Z.; Atef, M.; Yousef, K.; Lian, Y.; Wang, G.: A 61-nW level-crossing ADC with adaptive sampling for biomedical applications. IEEE Trans. Circuits Syst. II Express Briefs 66(1), 56-60 (2019)
41. Cox, J.R.; Nolle, F.M.; Fozzard, H.A.; Oliver, G.C.: AZTEC, a preprocessing program for real-time ECG rhythm analysis. IEEE Trans. Biomed. Eng. 15(2), 128-129 (1968)

42. Deepu, C.J.; Heng, C.H.; Lian, Y.: A hybrid data compression scheme for power reduction in wireless sensors for IoT. IEEE Trans. Biomed. Circuits Syst. 11(2), 245-254 (2017)

43. Zigel, Y.; Cohen, A.; Katz, A.: The weighted diagnostic distortion (WDD) measure for ECG signal compression. IEEE Trans. Biomed. Eng. 47(11), 1422-1430 (2000)

44. Mamaghanian, H.; Khaled, N.; Atienza, D.; Vandergheynst, P.: Compressed sensing for real-time energy-efficient ECG compression on wireless body sensor nodes. IEEE Trans. Biomed. Eng. 58(9), 2456-2466 (2011)

45. Jalaleddine, S.; Hutchens, C.G.; Strattan, R.D.; Coberly, W.: ECG data compression techniques-a unified approach. IEEE Trans. Biomed. Eng. 37(4), 329-343 (1990)

46. Chua, E.; Fang, W.C.: Mixed bio-signal lossless data compressor for portable brain-heart monitoring systems. IEEE Trans. Consum. Electron. 57(1), 267-273 (2011)

47. Chen, S.-L.; Lee, H.-Y.; Chen, C.-A.; Huang, H.-Y.; Luo, C.-H.: Wireless body sensor network with adaptive low-power design for biometrics and healthcare applications. IEEE Syst. J. 3(4), 398-409 (2009)

48. Chen, S.-L.; Wang, J.-G.: VLSI implementation of low-power cost-efficient lossless ECG encoder design for wireless healthcare monitoring application. Electron. Lett. 49(2), 91-93 (2013)

49. Deepu, C.J.; Lian, Y.: A joint QRS detection and data compression scheme for wearable sensors. IEEE Trans. Biomed. Eng. 62(1), 165-175 (2015)

50. Chen, S.-L.; Tuan, M.-C.; Chi, T.-K.; Lin, T.-L.: VLSI architecture of lossless ECG compression design based on fuzzy decision and optimization method for wearable devices. Electron. Lett. 51(18), 1409-1411 (2015)

51. Chen, S.-L.; Tuan, M.-C.; Lee, H.-Y.; Lin, T.-L.: VLSI implementation of a cost-efficient micro control unit with an asymmetric encryption for wireless body sensor networks. IEEE Access 5, 4077-4086 (2017)

52. Polania, L.F.; Carrillo, R.E.; Blanco-Velasco, M.; Barner, K. E.: Compressed sensing based method for ECG compression. In: Proceedings of IEEE International Conference on Acoustics, Speech and Signal Processing (ICASSP), pp. 761-764 (2011)

53. Ieong, C.-I.; Li, M.; Law, M.-K.; Mak, P.-I.; Vai, M.I.; Martins, R.P.: A $0.45 \mathrm{~V} \mathrm{147-375} \mathrm{nW} \mathrm{ECG} \mathrm{compression} \mathrm{processor} \mathrm{with} \mathrm{wavelet}$ shrinkage and adaptive temporal decimation architectures. IEEE Trans. VLSI Syst. 25(4), 1307-1319 (2017)

54. Kumar, R.; Kumar, A.; Pandey, R.K.: Beta wavelet based ECG signal compression using lossless encoding with modified thresholding. Comput. Electr. Eng. 39(1), 130-140 (2013)

55. Elgendi, M.; Mohamed, A.; Ward, R.: Efficient ECG compression and QRS detection for e-Health applications. Sci. Rep. 7(1), 1-16 (2017)

56. Zhou, J.; Wang, C.: An ultra-low power turning angle based biomedical signal compression engine with adaptive threshold tuning. Sensors 17(8), 1-12 (2017)

57. Abhishek, S.; Veni, S.; Narayanankutty, K.A.: Biorthogonal wavelet filters for compressed sensing ECG reconstruction. Biomed. Signal Process. Control 47, 183-195 (2019)

58. Abo-Zahhad, M.M.; Abdel-Hamid, T.K.; Mohamed, A.M.: Compression of ECG signals based on DWT and exploiting the correlation between ECG signal samples. Int. J. Commun. Netw. Syst. Sci. 7, 53-70 (2014)

59. Strang, G.; Nguyen, T.: Wavelets and Filter Banks. WellesleyCambridge Press, Wellesley (1996)

60. Daubechies, I.: Ten Lectures on Wavelets. SIAM, Pennsylvania (1992) 
61. Misiti, M.; Misiti, Y.; Oppenheim, G.; Poggi, J.M.: Wavelet Toolbox User's Guide. The MathWorks Inc., Natick (2009)

62. Padhy, S.; Sharma, L.; Dandapat, S.: Multilead ECG data compression using SVD in multiresolution domain. Biomed. Signal Process. Control 23, 10-18 (2016)

63. Tropp, J.A.; Gilbert, A.C.: Signal recovery from random measurements via orthogonal matching pursuit. IEEE Trans. Inf. Theory 53(12), 4655-4666 (2007)

64. Lu, L.; Yan, J.; de Silva, C.W.: Feature selection for ECG signal processing using improved genetic algorithm and empirical mode decomposition. Measurement 94, 372-381 (2016)

65. Schell, B.; Tsividis, Y.: A continuous-time ADC/DSP/DAC system with no clock and with activity-dependent power dissipation. IEEE J. Solid State Circuits 43(11), 2472-2481 (2008)
66. Tlili, M.; Ben-Romdhane, M.; Maalej, A.; Rivet, F.; Dallet, D.; Rebai, C.: Level-crossing ADC design and evaluation methodology for normal and pathological electrocardiogram signals measurement. Measurement 124, 413-425 (2018)

67. Jaw, F.-S.; Tseng, Y.-L.; Jang, J.-K.: Modular design of a long-term portable recorder for physiological signals. Measurement 43(10), 1363-1368 (2010)

68. Bera, P.; Gupta, R.: Hybrid encoding algorithm for real time compressed electrocardiogram acquisition. Measurement 91, 651-660 (2016) 